Draft Version June 4, 2019

Typeset using $\mathrm{LAT}_{\mathrm{E}} \mathrm{X}$ twocolumn style in AASTeX62

\title{
Scientific goals and in-orbit performance of the High-Energy Particle Detector on board the CSES satellite
}

P. Picozza, ${ }^{1}$ R. Battiston,${ }^{2}$ G. Ambrosi,${ }^{3}$ S. Bartocci,${ }^{1}$ L. Basara,${ }^{4}$ W. J. Burger,${ }^{5}$ D. Campana,${ }^{6}$ L. Carfora,${ }^{1}$ M. Casolino, ${ }^{7}$ G. Castellini, ${ }^{8}$ P. Cipollone,${ }^{7}$ L. Conti,${ }^{9}$ A. Contin, ${ }^{10}$ C. De Donato,${ }^{7}$ C. De Santis ${ }^{7}$ F.M. Follega, ${ }^{11}$ C. Guandalini, ${ }^{12}$ M. Ionica,${ }^{3}$ R. Iuppa, ${ }^{11}$ G. Laurenti, ${ }^{12}$ I. Lazzizzera, ${ }^{11}$ M. Lolli,${ }^{12}$ C. Manea, ${ }^{4}$ L. Marcelli, ${ }^{7}$ M. Martucci,${ }^{7}$ G. Masciantonio, ${ }^{7}$ M. Mergé,${ }^{7}$ G. Osteria,${ }^{6}$ L. Pacini,${ }^{8}$ F. Palma, ${ }^{1}$ F. Palmonari,${ }^{10}$ B. Panico, ${ }^{6}$ A. Parmentier,${ }^{7}$ L. Patrizit, ${ }^{12}$ F. Perfetto,${ }^{6}$ M. Piersanti,${ }^{7}$ M. Pozzato, ${ }^{12}$ M. Puel,${ }^{4}$ I. Rashevskaya,${ }^{4}$ E. Ricci, ${ }^{11}$ M. Ricci, ${ }^{13}$ S. Ricciarini, ${ }^{8}$ V. Scotti,${ }^{14}$ A. Sotgiu,${ }^{7}$ R. Sparvoli, ${ }^{1}$ B. Spataro, ${ }^{13}$ V. Vitale, ${ }^{7}$ P. Zuccon, ${ }^{11}$ AND S. ZOFFOLI ${ }^{15}$

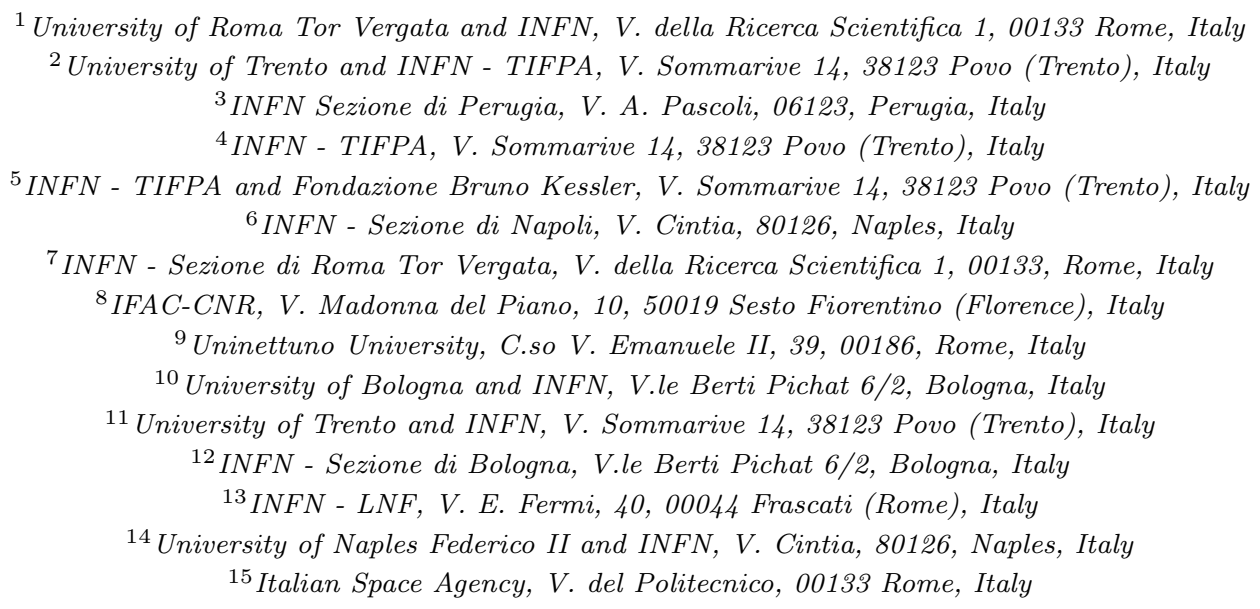

Submitted to ApJ Supplement

\begin{abstract}
The CSES satellite aims to monitor electromagnetic-, particle- and plasma perturbations in the ionomagnetosphere and inner Van Allen radiation belts, originated by electromagnetic sources external and internal to the geomagnetic cavity, cosmic rays and solar events. In particular, the objective of the space mission is to investigate lithosphere-atmosphere-ionosphere coupling mechanisms (including effects of lightning, earthquakes, volcanoes and artificial electromagnetic emissions) that induce perturbations of the top side of the ionosphere and lower boundary of the radiation belts.

To this purpose, the mission has been conceived to take advantage of a multi-instrument payload comprising nine detectors for the measurement of electromagnetic field components, plasma parameters and energetic particles, as well as X-ray flux. The Italian team participating in the CSES mission has built one of these devices, the High-Energy Particle Detector (HEPD), for high-precision observations of electrons, protons and light nuclei. During its trip along the orbit, and thanks to the large set of detectors operated on board, CSES completely monitors the Earth, acting as an excellent instrument for Space Weather.

The satellite was launched on Feb 2, 2018, with an expected lifespan of 5 years. This article describes the CSES mission with a particular focus on the HEPD apparatus and its in-flight performance.
\end{abstract}




\section{INTRODUCTION}

The lithosphere-ionosphere-magnetosphere coupling is a complex topic, due to a number of physical effects and interactions that occur between the Earth's surface and magnetosphere. Such interacting phenomena are mostly caused by natural events, tropospheric activity (e.g., lightning and volcanoes), and anthropogenic electromagnetic emissions. In addition, electromagnetic disturbances associated with seismic activity can induce ionospheric perturbations and possible precipitation of particles from the inner Van Allen belts. On the external side, the Sun causes regular and irregular variations of the geomagnetic parameters (by means of impulsive events, such as Coronal Mass Ejections - CMEs - and solar flares), and modulates the cosmic-ray flux.

The China Seismo-Electromagnetic Satellite (CSES), launched from the Jiuquan Satellite Launch Center in the Gobi Desert (inner Mongolia) on Feb 2, 2018 with a suite of advanced detectors, aims to shed new light on the spatial and temporal stability of the ionospheremagnetosphere transition zone, and to investigate disturbances induced by internal and external electromagnetic sources. CSES is part of a collaboration program between the China National Space Administration (CNSA) and the Italian Space Agency (ASI) (Shen et al. 2018). The mission has been developed by the China Earthquake Administration (CEA) and the Italian National Institute for Nuclear Physics (INFN), and includes several Chinese, Italian and Austrian universities and research institutes. CSES is the first element of a multi-satellite monitoring system, including several missions scheduled for the next few years, designed to investigate the top side of the ionosphere by means of the most advanced techniques and equipment, and able to gather worldwide data of the near-Earth electromagnetic environment.

CSES mission is monitoring the solar impulsive activity, such as the Solar Energetic Particle (SEP) emission and cosmic-ray solar modulation, by detection of protonand electron fluxes from a few $\mathrm{MeV}$ up to hundreds of $\mathrm{MeV}$. These measurements will provide an extension of particle spectra down to very low energies in the ongoing $24^{\text {th }}$ solar cycle, currently monitored at higher energies by experiments such as PAMELA (Adriani et al. 2017) and AMS-02 (Aguilar et al. 2013). CSES data will help analyze the temporal correlation between seismic events and the occurrence of both electromagnetic perturbations in the upper ionosphere and Van Allen particle precipitations, thus joining the mainstream of studies about lithosphere-atmosphere-ionosphere coupling mechanisms.
Early results on this topic were obtained by analyzing data from both ground- and space experiments mainly focusing on cosmic-ray observations, not specifically conceived for the investigation of seismo-electromagnetic phenomena. More recently, the robust development of small, low Earth orbit (LEO) satellites, such as CSES, is facilitating the study of seismo-associated electromagnetic phenomena by means of in-situ measurements of the induced ionospheric anomalies. DEMETER (Detection of Electro-Magnetic Emissions Transmitted from Earthquake Regions (Lagoutte et al. 2006; Cussac et al 2006)), a French microsatellite placed in a polar orbit at $700 \mathrm{~km}$, and relying on a set of payloads designed for multi-parametric measurements, has been the first mission specifically designed for this kind of investigation. CSES mission is planned to drive the pioneering experience of DEMETER forward through the collection of a long time series of data, which will help discern ionospheric disturbances caused by natural terrestrial events, such as earthquakes and volcanic eruptions, from the background induced by solar activity and anthropogenic sources. Moreover, the newborn CSES data center will contribute to provide the international scientific community with a multi-parameter observation sharing-service.

The Italian contribution to the mission includes the design and construction of HEPD, which is devised to detect electrons in the energy range between 3 and 100 $\mathrm{MeV}$, and protons between 30 and $200 \mathrm{MeV}$, as well as light nuclei (Ambrosi et al. 2018). The Space Research Institute (IWF) of the Austrian Academy of Sciences (AW) and the Institute of Experimental Physics (IEP) of the Graz University of Technology contribute to one of the magnetometers of the mission.

The article is organized as follows: in Section 2 an overview of the CSES mission is presented, while in Section 3 we discuss the scientific objectives of the mission, with special emphasis on those specific to HEPD. Section 4 is dedicated to the description of the High-Energy Particle Detector. Section 5 describes HEPD Qualification Tests, including tests with particle beams. In Section 6 we report details regarding the data Ground Segment of the HEPD instrument. Finally, Section 7 is devoted to the presentation of the in-orbit performance of HEPD after one year of flight. We draw our conclusions in Section 8. Technical details about the HEPD instruments and its electronics are reported in the Appendix.

\section{CSES MISSION OVERVIEW}

The CSES satellite, based on the Chinese 3-axis stabilized CAST2000 platform, moves along a sun- 

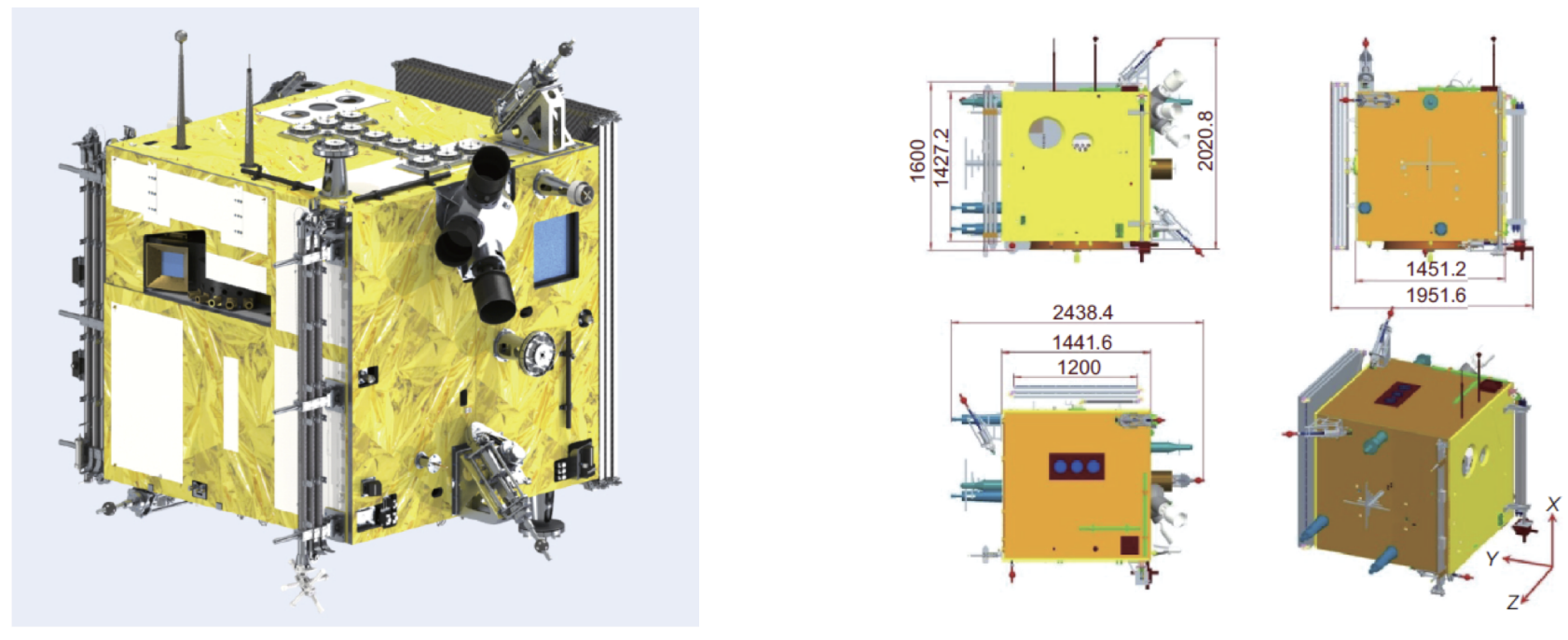

Figure 1. Layout of the CSES satellite with stowed solar panel and booms.

\begin{tabular}{l|l|l}
\hline \hline Platform & Mass & $\simeq 700 \mathrm{~kg}$ \\
\hline Orbit & Type & Sun-Synchronous \\
\hline & Altitude & $507 \mathrm{~km}$ \\
\hline & Inclination & $97^{\circ}$ \\
\hline & Period & $94 \mathrm{~min}$ \\
\hline & Local time descending node & $14: 00$ \\
\hline Data Transmission & Revisit period & 5 days \\
\hline & Dowd & $\mathrm{X}$ \\
\hline TMTC/OBDH & Mass Memory Size & $120 \mathrm{Mbps}$ \\
\hline & Uplink rate & $160 \mathrm{Gbit}$ \\
\hline AOCS & Downlink rate & $2000 \mathrm{bps}$ \\
\hline & Pointing accuracy & $16384 \mathrm{bps}$ \\
\hline & Knowledge accuracy & $\leq 0.1^{\circ}(3$-axis, $3 \sigma)$ \\
\hline Life Span & Stabilization accuracy & $\leq 0.03^{\circ}(3$-axis, $3 \sigma)$ \\
\hline \hline
\end{tabular}

Table 1. Main specifications and orbit parameters of the CSES satellite.

synchronous orbit at $507 \mathrm{~km}$ of altitude, with a $97^{\circ}$ inclination and a periodic 5-day ground track. CSES main body, in launch configuration, has dimensions 145 $\mathrm{cm}(\mathrm{Y}) \times 144 \mathrm{~cm}(\mathrm{Z}) \times 143 \mathrm{~cm}(\mathrm{X})$ (see figure 1$)$, which increase after the deployment of the solar panel and the booms.

The satellite includes several platform subsystems, such as the Attitude and Orbit Control (AOC), the OnBoard Data Handling (OBDH), the Tracking, Telemetry and Command (TTC), the Power Supply (composed of an $80 \mathrm{Ah} \mathrm{Li-ion} \mathrm{battery} \mathrm{and} \mathrm{solar} \mathrm{panels),} \mathrm{and} \mathrm{the} \mathrm{Ther-}$ mal Control subsystems. Table 1 summarizes the main specifications of CSES.
The AOC makes use of Earth-oriented 3-axis stabilization; attitude sensors (3 star trackers, 2 groups of gyros and 1 digital sun sensor) are used to measure the attitude, reaction wheel and magnetic torque in order to maintain the zero-momentum control. To reduce any interference on the scientific payloads from solar panel rotation or AOC adjustments, the satellite selects two working regions: 1) the payload working zone at latitudes between $-65^{\circ}$ and $+65^{\circ}$, and 2) the platform adjustment zone at latitudes $>+65^{\circ}$ or $<-65^{\circ}$, where the payloads stop working. When in orbit, the $\mathrm{X}$ axis of the satellite is oriented according to the velocity vector, while the $\mathrm{Z}$ axis points to the nadir. The solar panel located on one side of the satellite can be rotated around 
the $\mathrm{Y}$ axis at latitudes $>+65^{\circ}$ and $<-65^{\circ}$ in order to optimize the satellite power budget.

\subsection{On-board Scientific Payloads}

CSES hosts the following scientific payloads:

- a High-Precision Magnetometer (HPM) including a coupled dark-state scalar magnetometer and a fluxgate magnetometer for measuring the lowfrequency range, intensity, and three components of the magnetic field, respectively;

- a Search-Coil Magnetometer (SCM) for measuring the three components of the magnetic field at higher frequency;

- an Electric Field Detector (EFD) including 4 probes installed on as many booms, aimed at the measurement of the three components of the electric field;

- two instruments for plasma investigation, i.e., a Plasma Analyzer Package (PAP) and a Langmuir Probe (LP) in order to observe ion- and electron density and temperature, ion drift velocity and plasma composition;

- a GNSS Occultation Receiver and a threefrequency (VHF/UHF) beacon transmitter for studying the ionospheric plasma profile;

- two particle detectors, the Chinese High-Energy Particle Package (HEPP) and the Italian HighEnergy Particle Detector (HEPD), for measuring high-energy charged particles and X-ray flux.

Figure 2 shows the position of the payloads on board CSES.

\section{SCIENTIFIC GOALS OF CSES MISSION}

CSES mission has been designed to achieve two main scientific objectives: 1) to improve knowledge of the near-Earth electromagnetic environment (specifically, of the lithosphere-atmosphere-ionosphere coupling mechanisms, as well as the anthropogenic impact on the ionosphere), and 2) to better understand some phenomena of the solar-terrestrial interactions and cosmic rays in the Space-Weather context.

Near-Earth electromagnetic, plasma and particle environments are affected by broadband electromagnetic emissions due to lightning, whistlers, TLEs (Transient Luminous Events) and other phenomena connected to tropospheric activity, to anthropogenic sources (such as Very Low Frequencies - VLF - navigation and communication transmitters, broadcasting stations, powerline harmonic radiation, etc.) and to seismic and volcanic activity. Earthquakes are among the most dangerous natural disasters. Very strong earthquakes occur approximately 20 times a year over the globe. An earthquake is a deformation-, fracture-, structure- and phase-transformation event, which suddenly releases a large amount of the elastic energy stored in the medium (Earth's lithosphere) and is accompanied by a substantial fraction of energy radiated as seismic waves.

In the preparation phase of seismic events, some seismo-electromagnetic perturbations have been observed on ground and in space. Models proposed to describe their generation mechanisms invoke physical effects such as piezoelectricity, piezomagnetism and electrokinetic processes. During their propagation through the crust, the higher frequencies of seismo-associated disturbances are attenuated, and only the low-frequency components up to VLFs are supposed to reach the Earth's surface and propagate further into the nearEarth space [see e.g (Molchanov \& Hayakawa 1998; Hayakawa et al. 2010; Biagi et al 2008; Popova et al. 2018)]. The interaction of these electromagnetic emissions with the ionospheric environment can cause different kinds of direct and indirect perturbations in the plasma component, as well as in the electromagnetic field and particles trapped in the Van Allen radiation belts.

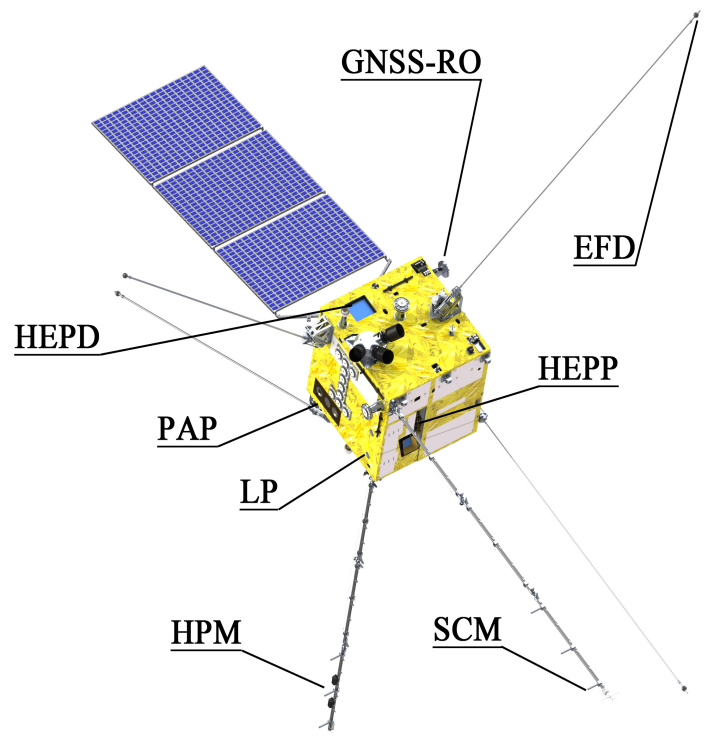

Figure 2. Position of the instruments on board CSES. The satellite is in orbit configuration, as shown by deployed solar panel and booms. 
Some LEO-satellite observations seem to confirm the above scenario. Pre-seismic changes of electric and magnetic fields (Molchanov et al. 1993; Parrot 1994, 1995), and of temperature and density of the ionospheric plasma (Parrot \& Mogilev 1989; Chmyrev et al. 1997; Yiyan et al. 2013), have been observed from a few minutes to several hours (2-6 hr) prior to earthquakes of moderate or strong magnitude. The most reliable results have been obtained by the analysis of DEMETER data, which include observations of some ionospheric perturbations related to earthquakes (Bertello et al 2018). Particularly relevant is the finding of an electromagnetic emission with a peculiar frequency of $333 \mathrm{~Hz}$ two days before the 6.3 Mw earthquake of L'Aquila in 2009.

The second main objective of CSES mission is to improve our knowledge of the solar-terrestrial interaction and cosmic-ray physics. Earth's geomagnetic cavity is a dynamic system generated by the effect of the solar wind and geomagnetic field, and structured in several macro-regions, such as the ionosphere, magnetosphere and Van Allen belts. They are affected by stationary and transient variations of their spatial and temporal composition and extension on local- and global scale. CSES follows many other missions, such as SAMPEX, THEMIS, LANL-GEO, GOES, aimed to investigate the composition of the Van Allen belts, the radiation-belt transport-, acceleration-, and loss mechanisms. Other missions, such as PAMELA and AMS-02, are investigating cosmic-ray physics. The CSES satellite is flying at altitudes comparable to, or lower than, the ones spanned by the other above-mentioned missions, and can detect particles in an intermediate energy range between those spanned by missions devoted to investigate radiation belts and those conceived for studying cosmic rays; in figure 3 a comparison between PAMELA galactic protons and electrons is shown, with superimposed the energy window of CSES/HEPD. This allows CSES to complement and integrate data from other missions. Specific goals will be described in detail in the following paragraphs.

\subsection{Specific goals of the High-Energy Particle Detector}

In the framework of CSES mission, HEPD has been built in order to increase our understanding of cosmic rays, to improve knowledge of some magnetospheric processes, to investigate the spatial and temporal stability of the inner Van Allen belts, and to study the impact of the natural electromagnetic emissions (in particular those due to seismic and volcanic activities) on the ionomagnetosphere transition zone.

\subsubsection{Cosmic-Ray and Solar studies}
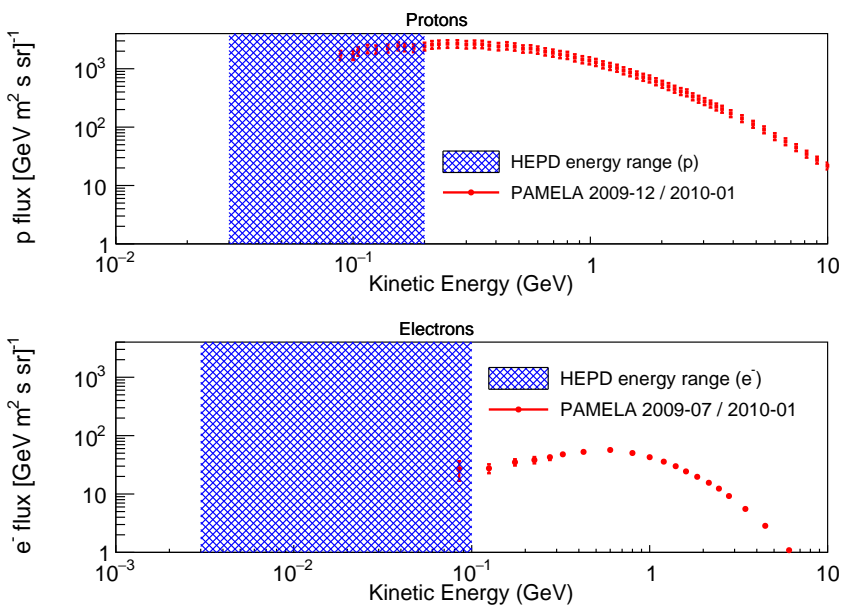

Figure 3. Comparison between PAMELA protons (upper panel) and PAMELA electrons (bottom panel). Data from (Adriani et al. 2013) and (Adriani et al. 2015a), respectively. Blue box refers to the energy range of CSES/HEPD.

For long-term space missions like CSES, the measurement of cosmic-ray particles needs to be related to the ever-changing environment in which the detector operates. The heliosphere, i.e. the region shaped by the presence of the solar wind flowing from the upper atmosphere of the Sun (the corona), presents different levels of variability, some of them still poorly understood. These changes in the solar-wind parameters (i.e., density, flow velocity, temperature, etc.) lead to the appearance of dynamic phenomena on many spatial and temporal scales (Balogh et al. 2008).

From launch to the scheduled end of mission, HEPD will be operative during the very last part of solar cycle 24 and the subsequent minimum of cycle 25 , offering ideal conditions to study mechanisms of propagation of cosmic rays inside the heliosphere. Such a quiet environment, in fact, could help obtaining an undisturbed description of all four major acceleration/transport factors in the Parker equation (Parker 1965): convection, particle drift (caused by gradients and curvatures in the heliospheric magnetic field), diffusion and adiabatic energy changes.

On such long time scales, the observation of electron and proton energy spectra, as well as their variations during one or more solar cycles (solar modulation), will be extremely helpful to understand the hierarchy of the processes that dominate the propagation of particles in the magnetized plasma. For example, many drift models (Potgieter 2014) predict a clear charge-sign dependence for the modulation of cosmic rays, increasing the importance of electron/proton joint measurements with high precision and high statistics. Other models predict that the diffusion coefficients may depend on energy in 
a much stronger way that previously expected. The role of these observations could gain further value, should the $25^{\text {th }}$ solar minimum be unusual like the $23^{r d}$, thus offering a unique chance to study such mechanisms in an unconventional situation. Low-energy data from HEPD could be also used to produce a full three-dimensional model (Potgieter et al. 2014) based on the numerical solution of Parker equation.

Solar modulation aside, every solar cycle presents a wide variety of shorter-timescale transients coming from the Sun, called solar particle events (SPEs). These events, which produce bursts of high-energy particles (SEPs), are rare during the minimum phase of solar activity, but the acceleration and propagation of such particles can be studied with lower uncertainty, usually caused by a turbulent heliosphere (which is typical of phases of maximum solar activity). The powerful Xclass event of Dec 6, 2006 (Struminski \& Zimovetz 2010), together with the Ground Level Enhancement (GLE) of Dec 13-14, 2006, represents a perfect example (Adriani et al. 2011).

During maximum activity, SEPs become more frequent, and a wide variety of data can be accumulated and compared, grouping different events according to some of their features (duration, spectral index, rollover energy, intensity, region of occurrence, etc.). Expected to be a > 5-year mission, CSES could hopefully register a large number of solar events in a range of energy (3-100 MeV for electrons and 30-200 MeV for protons) that lies between the in-situ observations by instruments like ACE, STEREO, GOES, etc. (Lario et al. 2013), and the high-energy data from neutron monitors on ground (Storini et al. 2005), thus filling the void left by PAMELA (Adriani et al. 2017, 2015b).

The $>30 \mathrm{MeV}$ threshold for protons, together with the spanning of latitudes between $\pm 65^{\circ}$, allows for particle detection in regions where the geomagnetic cutoff is considerably low, and even energetic particles from weak solar events can be measured.

The long period of data-taking will permit the collection of information about different categories of solar events, for example giving an answer to the question whether GLE and non-GLE events are just a different manifestation of the same mechanism with different energy, or two completely separate classes of events. The study of the relation between the spectral index and roll-over energy of a solar event could also shed light on the acceleration process that took place. Indeed, whether the Sun accelerates particles at low altitudes through magnetic reconnection, or at higher regions of the corona through coronal-mass-ejection (CME) driven shocks, is still a matter of study, given the complexity of the modifications induced by propagation phenomena across the interplanetary space (Mathews \& Venkatesan 1990). Concerning electrons, they are accelerated and released during large, gradual SEP events, reaching energies of tens of MeV (Haggerty \& Roelof 2009). Anyway, they are not directly measurable because of the concurring contribution of the small fraction escaping the corona and Bremsstrahlung-loss phenomena, combined with the distance from the source.

Medium-term transients, such as Forbush decreases (Forbush 1958), which are caused by a CME hitting Earth and shielding galactic particles that come from outside the magnetosphere, is another possible topic of interest. Large CMEs heavily affect the geomagnetic cutoff (Adriani et al. 2016), shrinking the portion of the magnetosphere that faces the Sun, and allowing more particles to precipitate at lower latitudes. The HEPD energy range is well suited to follow the evolution of such decreases, giving information about the magnitude of the decrease itself and recovery time of the galactic particles to normal conditions.

Scientific goals aside, CSES mission fits in a period when the issues concerning Space-Weather are being recognized as urgent, thus earning a chance to serve as a space monitor of the vicinity of the Earth, and to help develop further counteractions against possible dangerous solar phenomena.

\subsubsection{Stability of the Van Allen belts}

The Van Allen belts are zones of energetic charged particles, which are trapped in the Earth's magnetic field. There are two such belts (inner and outer) that extend from an altitude of about $1000 \mathrm{~km}$ up to about $65000 \mathrm{~km}$ above the Earth's surface. They are mainly composed of energetic electrons $(1 \mathrm{MeV}<\mathrm{E}<50 \mathrm{MeV})$ and protons $(10 \mathrm{MeV}<\mathrm{E}<1 \mathrm{GeV})$. The motion of trapped particles is assumed to be a superposition of three periodic motions: a gyration around the local magnetic field lines, a bouncing along field lines between conjugate mirror points in the northern and southern magnetic hemispheres, and a longitudinal drift around the Earth (see figure 4). The mirror points are the positions for which the (pitch) angle between the particle-velocity vector and the magnetic-field line takes the value $90^{\circ}$. At the mirror points, the particle's velocity-component parallel to the magnetic field reaches zero, and the particle reverses its motion. However, if the mirror point is located below the top of the atmosphere, the particle can be scattered by residual atoms of rarefied air, lose energy and get lost.

The outer and inner Van Allen radiation belts are extremely variable in composition, expecially when pow- 


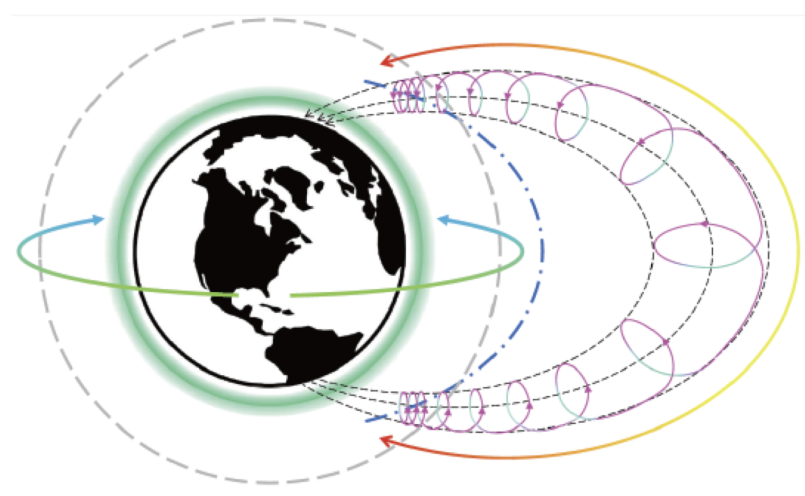

Figure 4. Schematic representation of the particle trapped by the geomagnetic field: geomagnetic-field lines (black thin dashed lines); gyromotion of trapped particles along the geomagnetic-field lines (cyan-magenta curly line); bouncing between the conjugate points (red-yellow line); West-East (East-West) longitudinal drift of the negative (positive) particles (green-blue lines); lower boundary of the inner radiation belt (dot-dashed blue line); ionosphere (green halo); low-altitude satellite orbit (grey dashed line).

erful SEPs encounter the magnetic-field lines (Adriani et al. 2016; Piersanti et al. 2017). These kinds of interactions (Van Allen \& Gangnes 1950) between the cosmic radiation and magnetosphere generate a set of particles called albedo (upward direction), which can be further identified either as re-entrant (if their trajectory is bent by the geomagnetic field, allowing them to remain trapped with a downward direction) or splash albedo (if they are able to escape the magnetosphere) (Treiman 1953). The former family comprises quasi-trapped and untrapped particles, depending on their confinement in the equatorial region below the inner Van Allen belt or not (Moritz 1972; Alcaraz et al. 2000), respectively.

New and accurate measurements of the high-energy $(>70 \mathrm{MeV})$ cosmic radiation at low Earth orbits have been reported in (Adriani et al. 2015c) as a function of energy and angle between the magnetic field and the direction of the incoming particle. Returning the incident particle direction, the HEPD instrument could measure, with the additional contribution from the ephemeris of the spacecraft and a tracing program based on numerical integration methods (Smart \& Shea 2000, 2005), albedo re-entrant protons up to $\sim 200 \mathrm{MeV}$, and provide material for comparison with previous experiments.

Furthermore, the geomagnetic field includes a peculiar region, the South Atlantic Anomaly (SAA), centered in Southeast America. The SAA (Kurnosova et al. 1962) is a region of lower geomagnetic field that causes the mirroring of the inner radiation-belt particles at lower altitudes, increasing the local particle flux. It is thus the region where the inner radiation belt makes its closest approach to the Earth's surface $(\sim 200 \mathrm{~km})$.

Being the modeling of this low-altitude radiation environment still incomplete, HEPD could bring new information by carrying on observations started with the PAMELA mission (>70 MeV for protons; between 2006 and 2009 (Adriani et al 2015d) in a different period, and extending the calculation to lower energies.

The CSES mission can have a crucial role in the investigation of the acceleration mechanisms, the global distribution and the variability of trapped particles in the Van Allen belts thanks to the multi-instrument payloads on board, which allow simultaneous measuring of the electromagnetic field [HPM and EFD instruments (Cheng at al. 2018; Diego et al. 2017a)], particles and plasma [PAP and LP (Diego et al. 2017b)]. Indeed, it is well known that the geomagnetic field changes under geomagnetic active conditions, giving rise to a redistribution of radiation-belt particles on both spatial and temporal scales.

The simultaneous and continuous monitoring of particle fluxes, electromagnetic fields and plasma composition is a requirement to determine how and where waves control the radiation-belt dynamics. Even though the largest changes in the geomagnetic field are due to the ring current variation during a geomagnetic storm (Kintner et al. 2002; Jordanova et al. 2006; Zaharia et al. 2006; Piersanti \& Villante 2016), also resonant waveparticle interactions strongly affect belt electrons. Indeed, the chorus can both accelerate relativistic electrons and make them precipitate, while the plasmaspheric hiss can control the location and dynamics of the "slot" region (Vellante et al. 2014,b). In this context, the HEPD payload on board the CSES satellite ensures the highest-quality measurement of charged particles below the inner Van Allen belts at energies intermediate between those detected by the RBSP-ECT (Radiation Belt Storm Probes - Energetic Particle, Composition, and Thermal Plasma) suite of the Van Allen Probes mission, and the energies of PAMELA and AMS-02 missions. Therefore, HEPD data can extend and complement the observations from Van Allen Probes, as well as those performed by PAMELA and AMS-02 missions.

In addition, HEPD data can be used to study ElectroMagnetic Ion Cyclotron (EMIC) emissions (Omura et al 2010, and reference therein). Ion cyclotron instability driven by the anisotropic distribution of ringcurrent energetic ions during magnetic storms can generate extremely-low-frequency (ELF) waves in the equatorial region (Lorentzen et al. 2000; Summers \& Thorne 2003; Meredith et al. 2003). These emissions can be observed at CSES altitudes by on-board electromagnetic 
instruments in the ELF range (from fractions of $\mathrm{kHz}$ up to a few $\mathrm{kHz}$ ), and be analyzed in order to clarify their physical mechanisms.

\subsubsection{Investigation of the coupling between seismicity and Van Allen local instability}

Anomalous fluxes of particles have been detected by some space experiments a few hours before the occurrence of earthquakes (Battiston \& Vitale 2013). These short-term particle bursts have been interpreted as due to the precipitation of particles from the inner belt as a result of interactions between seismo-electromagnetic emissions and Van Allen radiation-belt particles. Of course, seismicity is not the only possible explanation for particle bursts.

A possible interaction mechanism is the resonance between seismo-electromagnetic waves and particles with bouncing frequency in the same band (e.g., between ultra-low-frequency, ULF, waves and electrons of energy $>1 \mathrm{MeV}$ or protons of energy of the order of some tens of $\mathrm{MeV}$ ). When the wave frequency matches the particle bouncing frequency, the particle experiences the wave electric field $\mathrm{E}$ at every passage over the perturbed zone. This could produce a variation in the particle pitch angle, a lowering of its mirror points and the possible precipitation of the affected particle (Aleshina et al. 1992). Due to the standard longitudinal drift, also the precipitating particles continue to drift around the Earth along the same unperturbed L-shell ${ }^{1}$ where the interaction occurred, thus creating a particle wave that propagates around the Earth, with electrons drifting eastward and protons drifting westward. This wave gets slowly damped due to energy losses in the residual atmosphere, and spreads in space due to the dispersion of particle angular velocity. Due to the drift of trapped particles around the Earth, any particle burst of seismic origin can be observed not only above the epicenter but also at any longitude where the satellite crosses the disturbed L-shell. Therefore, if the seismic nature of particle bursts will be confirmed, and their statistical significance assessed, these three factors (i.e., the particle drift in the same L-shell, the temporal behavior of the damped particle wave and the opposite drifts of electrons and protons) would, in principle, allow to re-construct the geographic zone of the incipient earthquake.

It is worth remarking that on-ground and ionomagnetospheric electromagnetic perturbations possibly

\footnotetext{
1 The L-shell is a parameter describing a particular set of Earth's magnetic field lines. In particular, it describes field lines crossing the Earth's magnetic equator at a number of Earth radii equal to the L-value.
}

induced by seismicity must be carefully distinguished from the large background caused by geomagnetic natural fluctuations and electromagnetic emissions of anthropogenic origin. To this purpose, on the one hand, data collected during geomagnetic perturbed periods (selected by means of geomagnetic indices such as $\mathrm{Kp}$, Dst, Ap, etc.) cannot be used for statistical analysis. On the other hand, ground-based signals from radionavigation and communication VLF transmitters can interact with trapped particles around the geomagnetic equator, and powerful transmitters of HF broadcasting stations can induce ionospheric heating phenomena due to change in the local temperature and density of plasma components. Finally, tropospheric electromagnetic emissions due to lightning and whistlers can generate characteristic electromagnetic phenomena in the top side of the ionosphere. In these circumstances, data cannot be used for statistical analyses too.

The measurement of high-energy charged particle fluxes has been obtained on board various spacecrafts: the MIR orbital station $\left(20 \mathrm{MeV}<\mathrm{E}_{e}<200 \mathrm{MeV}\right)$, METEOR-3 $\left(\mathrm{E}_{e} \leq 30 \mathrm{MeV}\right)$, GAMMA $\left(\mathrm{E}_{e} \geq 50 \mathrm{MeV}\right.$ and $\mathrm{E}_{e} \leq 15 \mathrm{MeV}$ ) by means of different instruments. Such fluxes have been processed and analyzed in order to search for temporal and spatial correlation between particle bursts and strong earthquakes (Aleshina et al. 1992; Aleksandrin 2003; Sgrigna et al. 2005). In the analyses, sharp short-term increases of particle count rates, from tens of seconds to a few minutes, were identified as particle bursts if their probability exceeded a given threshold cut with respect to the average value of the background.

In particular, (Aleksandrin 2003) performed a multiinstrument data analysis. Figure 5 shows the temporal correlation between particle bursts and a set of earthquakes with magnitude $\mathrm{M}>4$, after selection of satellite positions at $\mathrm{L}$-shell $<2$ (i.e., the near-equatorial region) and exclusion of the SAA region. The value of $\Delta \mathrm{T}$ was defined as:

$$
\Delta T=T_{E Q}-T_{P B},
$$

where $T_{E Q}$ and $T_{P B}$ are the times of occurrence of the earthquake and particle burst, respectively. The positive value of all the peaks $(2-5 \mathrm{hr})$ means that the particle bursts could play the role of short-term earthquake precursors. In the analysis, sharp short-term increases of particle count rates, from tens of seconds to a few minutes, were selected as particle bursts whenever the count rate exceeded the average value of the background by 4 standard deviations. (Aleksandrin 2003) also investigate the spatial correlation between bursts and earthquakes by monitoring the variation of temporal distributions, 

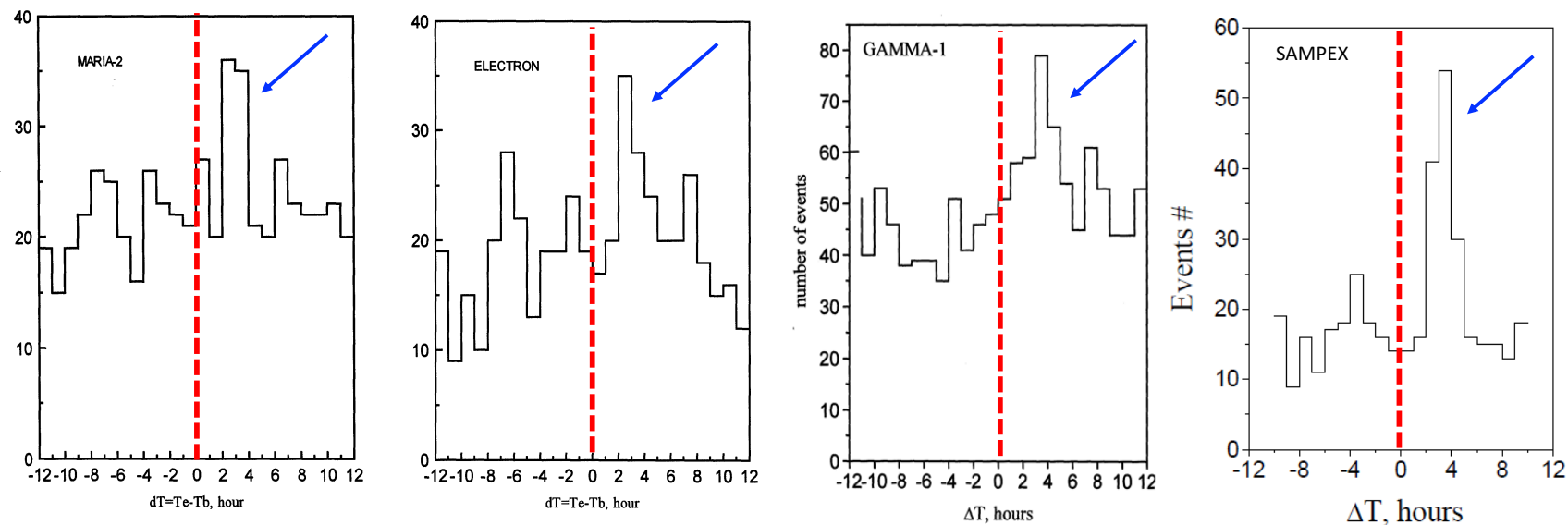

Figure 5. Histograms of time difference $\Delta T$ between the time of selected earthquakes and that of particle bursts obtained by MARIA, ELECTRON, GAMMA-1 and SAMPEX space missions. A positive value of the peak suggests that particle bursts precede earthquakes in time. Plots are from (Aleksandrin 2003).

using

$$
\Delta L=L_{E Q}-L_{P B}
$$

as an additional parameter. $L_{E Q}$ is the L-shell of the earthquake (i.e. the L-coordinate of the point at a certain altitude above the epicenter, which coincides with the altitude of the region by which the electromagnetic emission of seismic origin is captured in the geomagnetic field lines), and $L_{P B}$ is the L-shell of the satellite corresponding to the particle burst. The analysis shows that $\Delta \mathrm{T}$ distributions present no peaks when $\Delta L>0.5$, i.e., the L-shell of the earthquake significantly differs from that of the particle bursts, which is a result in support to correlation between particle precipitation and earthquakes.

Moreover, recent studies on low-energy electrons (E $>0.3 \mathrm{MeV}$ ) collected by the NOAA Polar Operational Environmental Satellites (POES) during 13 years (Battiston \& Vitale 2013) found a statistical temporal correlation between the occurrence of pairs of particle bursts and earthquakes of magnitude $\mathrm{M}>5$.

Starting from these premises, also the HEPD detector has been designed to investigate correlations between particle bursts and seismic activity. Indeed, the device has been conceived to detect electrons and protons in the energy range of interest, with wide angular acceptance (about 1 sr over the full energy range) and geometry factor large up to $300 \mathrm{~cm}^{2} \mathrm{sr}$ at the peak, that is at least 100 times larger than the geometric acceptance of DEMETER and at least 1000 times larger than the one of NOAA POES. HEPD will also be able to investigate the existence of bursts of protons and light nuclei possibly correlated to major earthquakes.

Although low-frequency seismo-associated electromagnetic emissions have been observed on ground close to earthquake epicenters, and in space by several satel-

lites before strong earthquakes, their pre-seismic nature and postulated role in the lithosphere-ionosphere coupling mechanisms is far from experimentally confirmed. Further studies on this topic are needed in order to understand the physical mechanisms of the above-mentioned correlations: earthquake forecast is not possible at present.

\section{THE HEPD INSTRUMENT}

The High-Energy Particle Detector, built by the Italian Limadou collaboration, is shown in figure 6, where two schematic views of the apparatus - with lateral and top panels removed - are shown.

From top to bottom, a particle entering the detector will cross:

- a tracking system made of 2 planes of doublesided silicon micro-strip sensors, suitable for the reconstruction of the incident-particle trajectory and the measurement of the energy loss per unit length, useful for particle identification;

- a trigger system consisting of one layer of plastic scintillator divided into six segments (paddles) and read out by photo-multiplier tubes, which generate an efficient trigger signal to synchronize data acquisition for the whole detector;

- a range calorimeter composed of a first section that comprises 16 planes of plastic scintillator on the top, and a layer of LYSO crystals on the bottom, suitable for the measurement of the energy deposition and range of impinging particles;

- an anti-coincidence (veto) system - including 5 (4 lateral and one bottom) plastic-scintillator planes read out by photo-multiplier tubes - which is used 

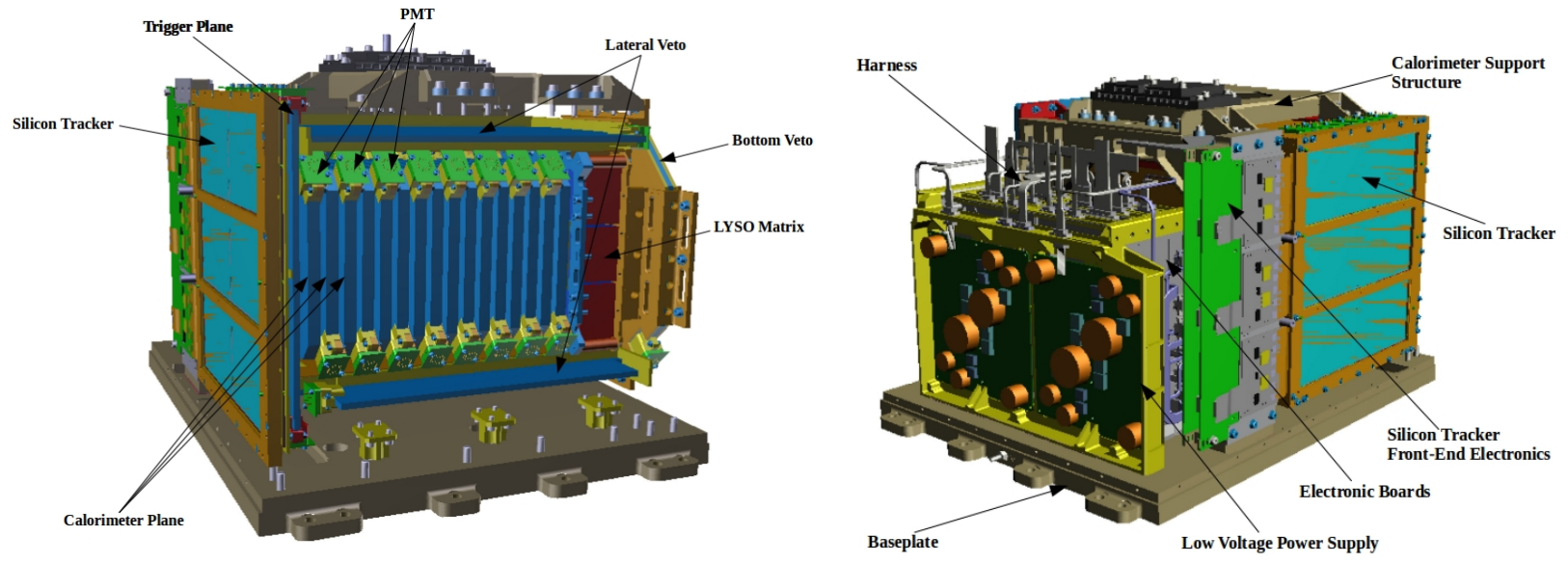

Figure 6. Schematic views of the HEPD electronics box and detecting units.

to detect particles that enter the apparatus from outside the acceptance, or particles that are not fully contained within the calorimeter;

On the lateral side of the sensitive detectors, two main sub-systems complete the whole instrument:

- an electronic sub-system made of four boards for trigger management, data acquisition, On-Board Data Handling (OBDH), slow control, and lowvoltage power distribution;

- a power supply sub-system composed of a board (Low-Voltage Power Supply) that generates two low-voltage power lines from the satellite power bus, and a system (High-Voltage Power Supply) that produces two high-voltage lines (up to $150 \mathrm{~V}$ and $1200 \mathrm{~V}$, respectively).

The HEPD detector is contained in an aluminum box with dimensions $40.36 \times 53.00 \times 38.15 \mathrm{~cm}^{3}$. The walls and base plate are made of milled aluminum panels, with the outside surface covered by a black anodized coating in order to assure a good thermal insulation. The detector, together with the power supply and electronics boxes, is fixed to the satellite cabin space, which provides the contact surface for heat dissipation. The total instrument mass is about $45 \mathrm{~kg}$; the power consumption depends on the status of HEPD, but is always lower than $30 \mathrm{~W}$.

Technical details about the characteristics of the subdetectors of the HEPD apparatus and its electronics are reported in the Appendix.

\section{HEPD MODELS AND QUALIFICATIONS TESTS}

Following the standard space procedures, four HEPD models were produced and fully integrated in the clean rooms at INFN laboratories of Roma Tor Vergata in
Rome (Italy). The Electrical Model (EM), including only electrical and transmission sub-systems, was used to validate the wire connections between the payload and satellite. The Structural and Thermal Model (STM), mechanically equal to the final instrument, but with dummy sensors and electronics instead of real ones, was constructed to validate structural and mechanical design, as well as thermal conductivity.

The Qualification Model (QM), identical to the flight detector, was used to qualify all the sub-systems by stress tests, calibrate the instrument, and assess its compliance with space requirements.

The QM underwent thermal-vacuum, pyroshock, vibration and thermal-cycling tests from May to August 2016. These tests took place at the SERMS Laboratory in Terni (Italy). Specifically, in June 2016 the HEPDQM was exposed, along its 3 directions, to pyrotechnic shocks with a shock frequency ranging from 600 to $4000 \mathrm{~Hz}$, and an acceleration up to $1000 \mathrm{~g}$. In June 2016, the Qualification Model successfully underwent sinusoidal and random vibration tests as well. Sinusoidal vibration tests were performed along the 3 axes in the frequency range from 20 to $100 \mathrm{~Hz}$ corresponding to a $12-\mathrm{g}$ acceleration.

The operational temperature requirement for HEPD is from $-10^{\circ} \mathrm{C}$ to $+35^{\circ} \mathrm{C}$, and it was extensively tested in both thermal- and thermal-vacuum chambers. In $\mathrm{Au}-$ gust 2016, the HEPD-QM was tested in a climatic chamber at ambient pressure: 25.5 temperature cycles were run from $-30^{\circ} \mathrm{C}$ to $+50^{\circ} \mathrm{C}$ with a $(3-5)^{\circ} \mathrm{C} / \mathrm{min}$ temperature rate of change and a dwell time of $\geq 4$ hours. The dwell time is the duration required for the detector temperature sensors to achieve stabilization. After that, about 6 temperature cycles were performed in a thermal-vacuum chamber between $-30^{\circ} \mathrm{C}$ and $+50^{\circ} \mathrm{C}$ at 


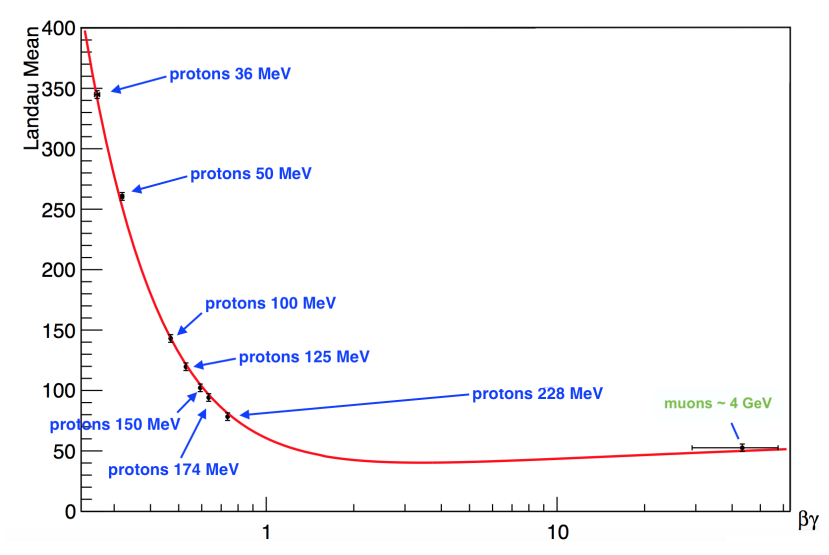

Figure 7. Calibration curve of one tracker ladder of HEPD. On the $\mathrm{Y}$ axis, the mean of the Landau distribution of the energy deposited by the beam-test particles at a given energy is reported. Also one point obtained by cosmic ray-muons is shown. The superimposed red line is the theoretical Bethe curve.

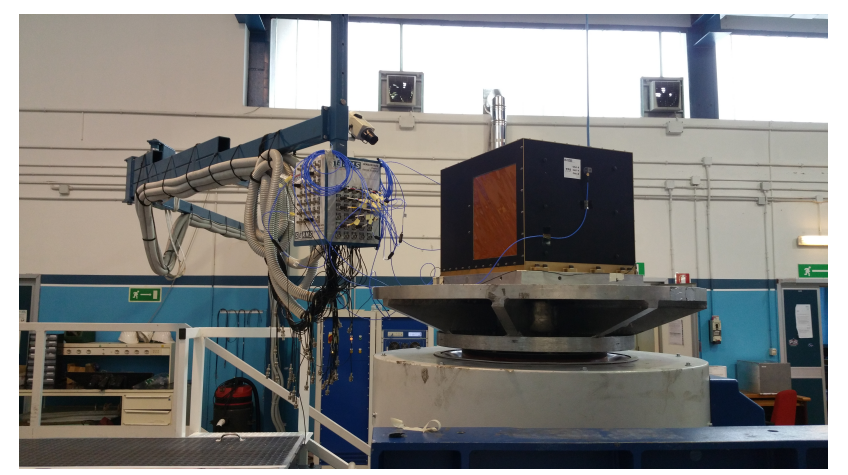

Figure 8. HEPD Flight Model on the shaker system at the SERMS facility in Terni (Italy) in October 2016.

a pressure $\leq 6.66 \times 10^{-3} \mathrm{~Pa}$. Nine temperature sensors were placed on the detector under test, eight internally and one externally. The chamber temperature gradients were $2^{\circ} \mathrm{C} / \mathrm{min}$ and $1^{\circ} \mathrm{C} / \mathrm{min}$ during the heating and cooling, respectively, and the dwell time was $\geq 4$ hours. Each cycle is composed of different steps corresponding to different operations for HEPD, such as data acquisition, calibration, stand-by or power off, in order to simulate in-flight procedures. During these operations, HEPD telemetry packets were continuously monitored to check the presence of anomalies in the detector, and in scientific data as well. The Electrical Ground Support Equipment (EGSE) was used to provide voltage to HEPD, and to send telecommands to the detector, in order to change status and configurations according to test requirements.

A space qualification campaign for the Flight Model (FM) followed, in the period October-November 2016.
Vibration tests (figure 8) were the first to be performed, in similar conditions (3 axis, 20-100 Hz, $8 \mathrm{~g}$ ) as for the HEPD-QM. During the thermal-cycling and thermalvacuum tests, fewer cycles (17.5 and 4.5, respectively) and less strong conditions (from $-20^{\circ} \mathrm{C}$ to $+45^{\circ} \mathrm{C}$ ) were required for the HEPD-FM. No structural damage and no loss of functionality or scientific performance were observed after the instrument qualification for space operations, therefore the HEPD-FM was definitely shipped to DFH Satellite Company, Ltd. in Beijing (China) in December 2016.

In January 2017, the stand-alone functionality of the instrument was successfully tested by means of its EGSE; then it was installed on CSES satellite at DFH Satellite Company, Ltd. in Beijing (China), as shown in figure 9. Random vibration- and thermal-vacuum tests were successfully repeated on board CSES satellite in February and April 2017, respectively, while magnetic cleanliness and aging tests were accomplished in May 2017.

During the final assembly phase, and before the final delivery to China, the HEPD-FM was tested under particle beams in different laboratories. At the INFNLNF Beam Test Facility (October 2016, Frascati, Italy) the instrument was exposed to electron beams of 30 , 60, 90 and $120 \mathrm{MeV}$, while at the Trento Protontherapy Center (November 2016, Trento, Italy) proton beams of $37,51,70,100,125,154,202$, and $228 \mathrm{MeV}$ were available. In addition, the instrument was exposed to the acquisition of cosmic rays (muons) in Tor Vergata clean rooms, Rome, Italy (November-December 2016). A very accurate calibration of the HEPD silicon tracker was performed with proton-beam test data at different energies. Figure 7 shows a very good agreement between experimental points (from proton-beam data) and theoretical expectation (red line) for energy loss in a ladder of the tracker.

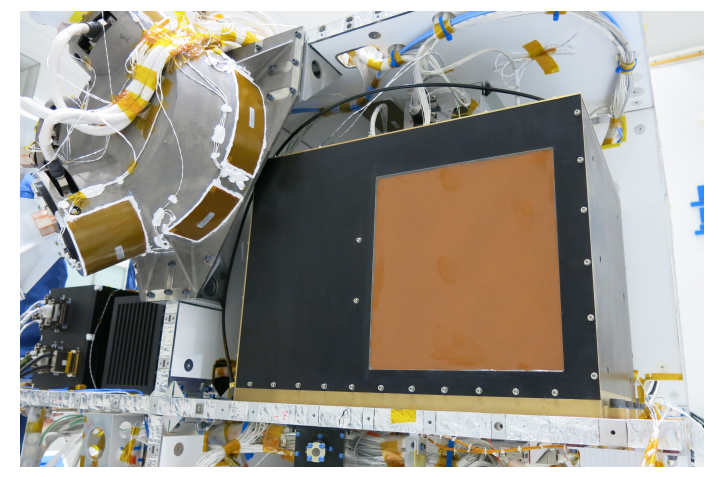

Figure 9. HEPD Flight Model installed on CSES satellite at DFH Satellite Company, Ltd. in Beijing (China) 


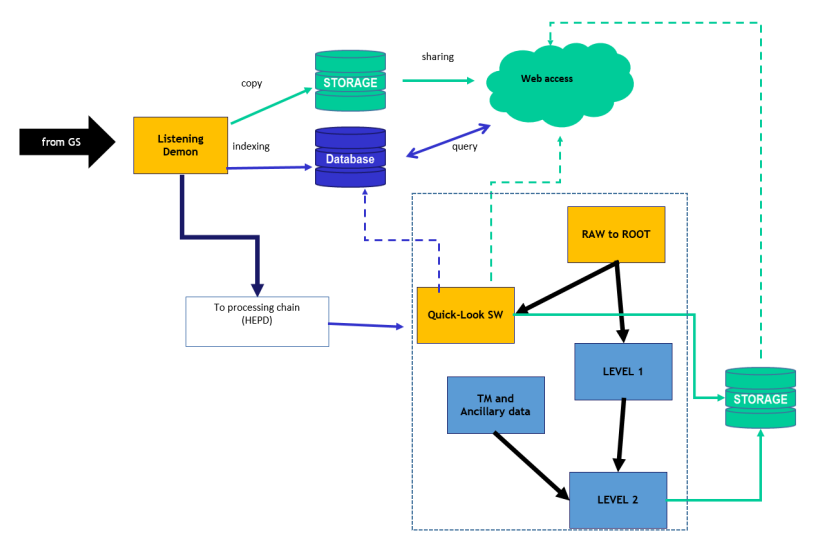

Figure 10. Scheme of data pipeline from satellite to web access and storage.

In 2018 the HEPD Qualification Model, once shipped back to Italy after the test campaign in China, was exposed to a beam at INFN-LNS laboratories in Catania (Italy). This additional test was aimed to further study HEPD performance, especially for particle charges greater than $Z=1$. The available beams were protons, helium, carbon, and oxygen at an energy of 62 $\mathrm{MeV} / \mathrm{amu}$. Analysis of data acquired during the Catania beam test is still in progress.

\section{HEPD GROUND SEGMENT}

HEPD data are transferred from the Chinese Ground Segment at the Institute of Crustal Dynamics of the China Earthquake Administration to a dedicated highavailability infrastructure installed at the Italian Space Agency Space Science Data Center.

High availability is achieved duplicating the processing servers, as well as the networking layer. The storage is accessed by redundant controllers, and the FreeNAS software ensures resilience of data on storage. Here, processing from raw data to calibrated (so-called "level2") data will be performed, and the infrastructure will also handle a bookkeeping database of the received/processed files, a quick-look database for monitoring the detector behavior. Once the data are transferred, an automatic pipeline removes the transmission frames, checks the integrity of the files, and transforms the raw data into ROOT format for further analysis.

In addition to HEPD data, also level2 data from the other scientific payloads on board CSES will be received, that is, magnetometers, the electric field detector, and plasma analyzers. The full level2 dataset collected in this infrastructure will be distributed to other institutions involved in data analysis.

Figure 10 represents the scheme of the data pipeline from satellite to web access and storage.
According to the data policy of the CSES collaboration, data will be public through a Chinese interface ${ }^{2}$. As for HEPD, proton and electron counting rates integrated over 1 second intervals and in different energy bins will be available in the database, together with orbital information.

\section{HEPD IN-ORBIT PERFORMANCE}

The CSES satellite was launched on Feb 2, 2018. On Feb 6, the HEPD instrument was switched on for the first time. The apparatus underwent the commissioning phase (February-July 2018), during which several onboard configurations were changed for testing purposes. Such procedures are crucial to guarantee optimal operational conditions throughout the foreseen 5 -year mission duration. In particular, in the commissioning phase we studied the stability of the electronics - especially that of PMT calibration pedestals - as well as the thresholds for the acquisition trigger, while implementing fine tuning of the trigger configuration as a function of in-flight particle rates.

A map of the trigger rate from May 14 to June 11, 2018, when HEPD operated in a continuous data acquisition mode and in the same stable configuration, is shown in figure 11. The condition for trigger generation is signal coincidence on the trigger plane $\mathrm{T}$ and the first three planes, P1, P2 and P3, of the calorimeter. The red spot around Brazil represents the South Atlantic Anomaly (SAA), in which the rate counter saturates at about $350 \mathrm{~Hz}$. In the bottom panel of the same figure, the trigger rate as a function of on-board time is reported. Red peaks refer to the passages over the South Atlantic Anomaly, where the proton belt is nearer to the Earth's surface, owing to the tilting of the magnetic axis of the planet, and causing the presence of a vast majority of trapped particles. On the other hand, the polar regions (marked as NP and SP, respectively, in the plot) present a markedly lower rate than the SAA, but higher than the equatorial portion (marked as EQ) of the Earth, since, at higher latitudes, the geomagnetic cutoff becomes smaller, thus allowing low-energy galactic particles (normally absent at lower latitudes) to contaminate the samples.

In addition to event selection in accordance to any single "trigger mask" (which can be changed by transmission of a dedicated command), the detector is also capable of counting particles that simultaneously transit across all other implemented trigger configurations, by means of several counters that act independently of the original trigger mask that was chosen. These counters

\footnotetext{
2 http://www.leos.ac.cn/Areweb_TH_system/data-getData
} 
allow for different particle selections, indirectly limiting the energy thresholds, and possibly giving an estimation of the energy dependence.

Figure 12 shows the count-rate map for:

- the trigger configuration $\mathrm{T}$; this is the configuration with the lowest energy threshold, which allows to capture low-energy particles in the outer Van Allen belt at latitudes larger than $+50^{\circ}$ and smaller than $-40^{\circ}$ (top picture);

- the trigger configuration $T \& P 1$, including the trigger plane and the first calorimeter plane (central picture);

- the trigger configuration $T \&(P 1|| P 2) \&(P 15|| P 16)$, including the trigger plane, and the first and last plane of the calorimeter (bottom picture),

respectively.
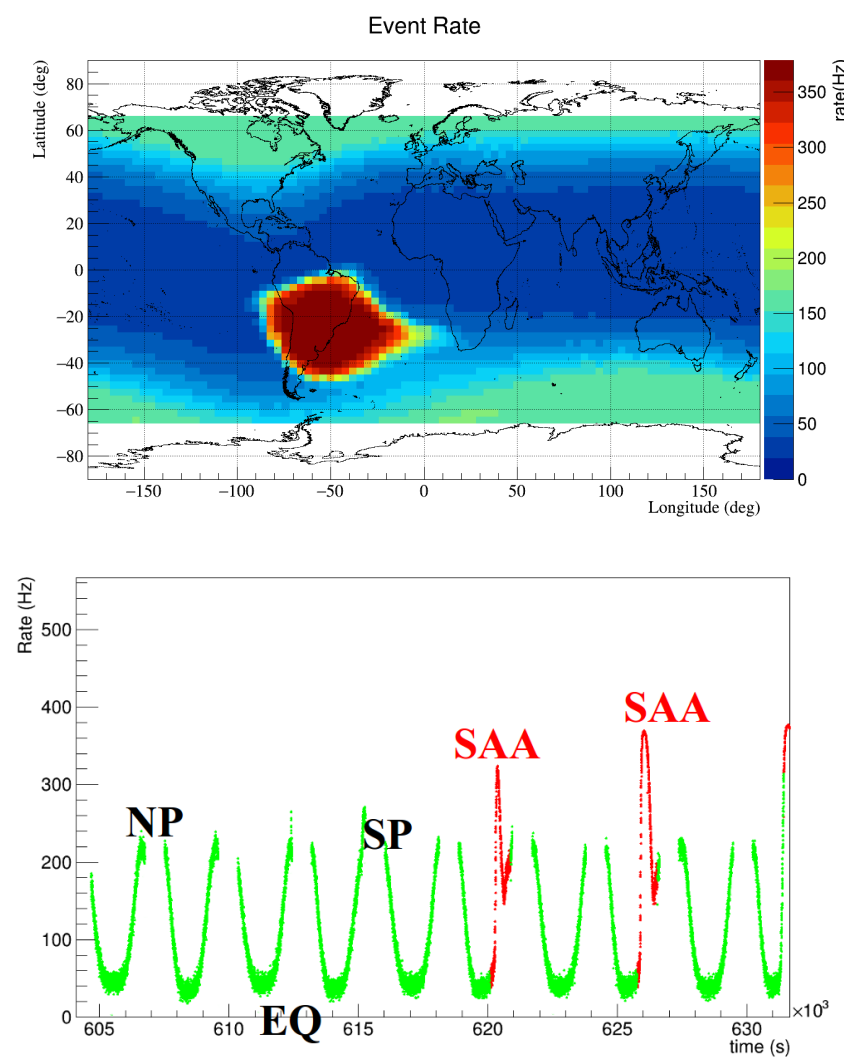

Figure 11. Top Panel: HEPD average event-rate map during the period from May 14 to June 11, 2018. The red spot around Brazil represents the South Atlantic Anomaly, in which the rate counter saturates at about $350 \mathrm{~Hz}$. Bottom Panel: HEPD trigger rate as a function of on-board time for a few orbits. Different regions are marked (namely, SAA for South Atlantic Anomaly, NP and SP for North and South Pole, respectively, and EQ for Equatorial Region).
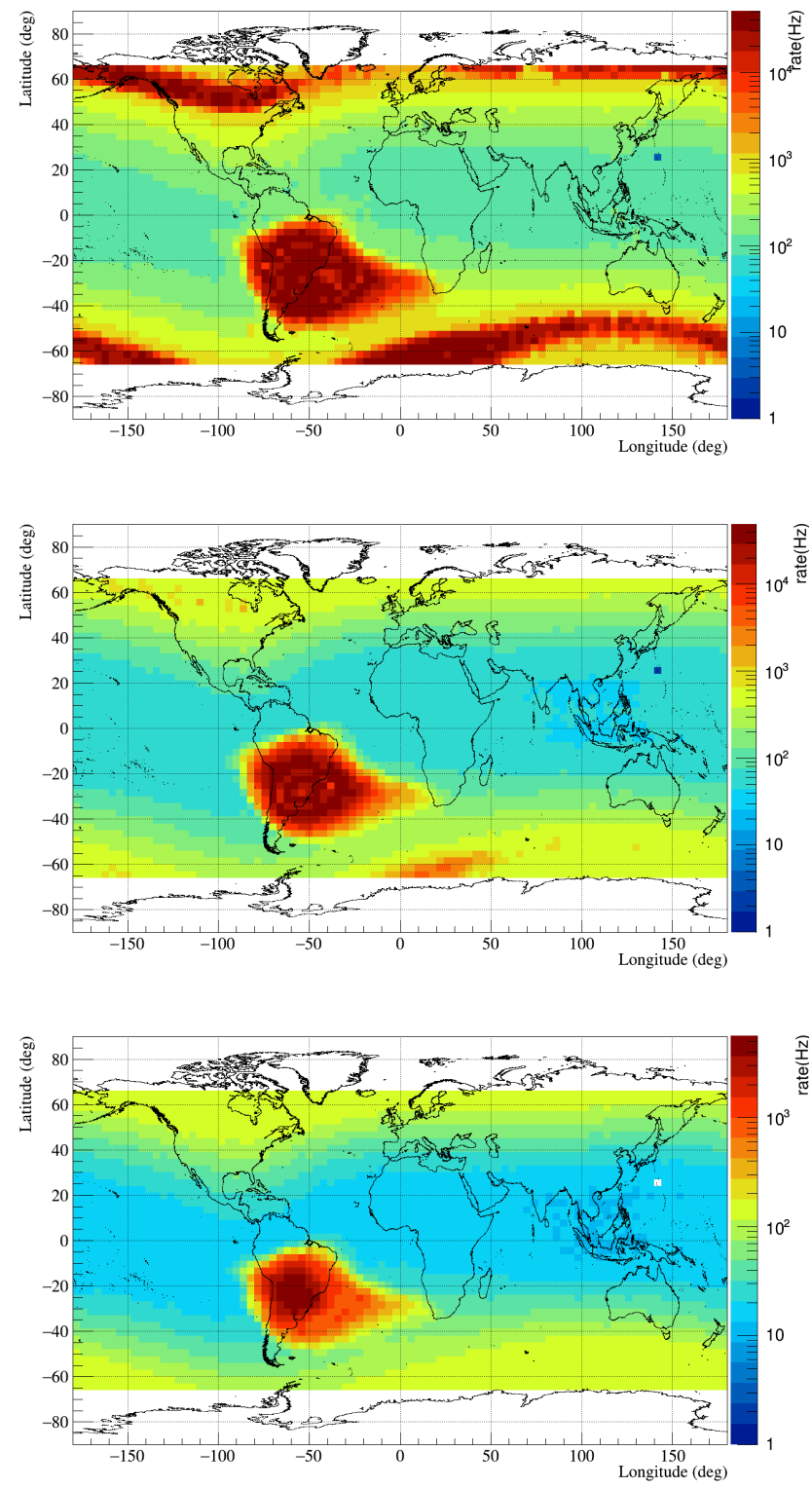

Figure 12. Top: Count-rate map for HEPD trigger plane. This trigger-mask configuration is the one with the lowest energy threshold, and consequently the most sensitive to lowenergy electrons from the outer Van Allen belt (red-shaded areas in the polar regions) and low-energy trapped protons in the South Atlantic Anomaly. Center: Count-rate map for the configuration including the trigger plane and the first plane of the calorimeter (T\&P1). Bottom: Count-rate map for the trigger configuration $T \&(P 1|| P 2) \&(P 15|| P 16)$.

Other intermediate trigger-configuration rate-meters are implemented in the detector, which could be extremely helpful in the definition of the best trigger mask to adopt on specific occasions. This could be the case of Solar Particle Events (SPEs), when a lowthreshold configuration could be useless due to the 


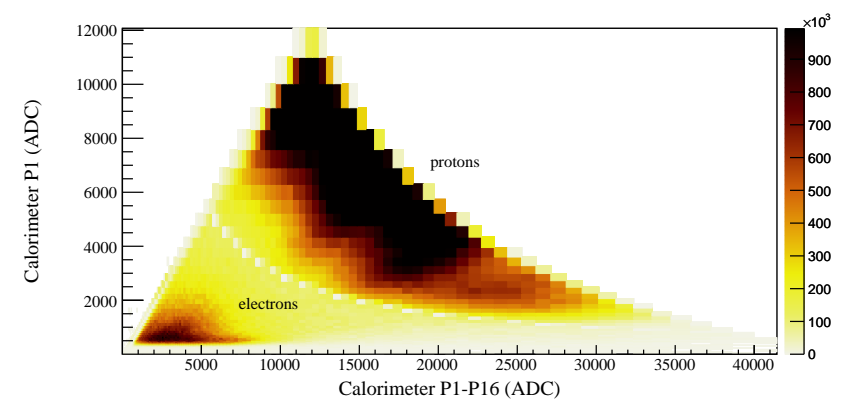

Figure 13. In-flight proton-electron identification by HEPD: energy released in the first plane of the calorimeter (P1) as a function of that released in the full calorimeter (P1 $+\ldots+P 16)$.

huge amount of triggering events involved, whereas a "deeper" configuration could prevent instrument saturation, providing a reliable detection of the solar component at the same time.

Particle identification is a rather easy task for HEPD, thanks to the large set of independent information coming from different detecting units. Figure 13 shows particle separation into protons and electrons during inflight detection, based on the information returned by the trend of the energy deposited in the first calorimeter plane $(\mathrm{P} 1)$ as a function of that released in the full calorimeter $(\mathrm{P} 1+\ldots+\mathrm{P} 16)$.

Figure 14 shows how HEPD can detect different particle populations according to satellite position (defined by the L-shell parameter) and energy detected: in the picture, particles trapped in both inner (SAA) and outer Van Allen belts can be appreciated, together with cosmic rays of galactic origin. Further, HEPD periodically crosses the SAA along its orbit, and can thus map its morphology by searching for trapped protons in that specific position of the geomagnetic field. Figure 15, for example, highlights the coherence between trappedproton geographical distributions inside the South At-

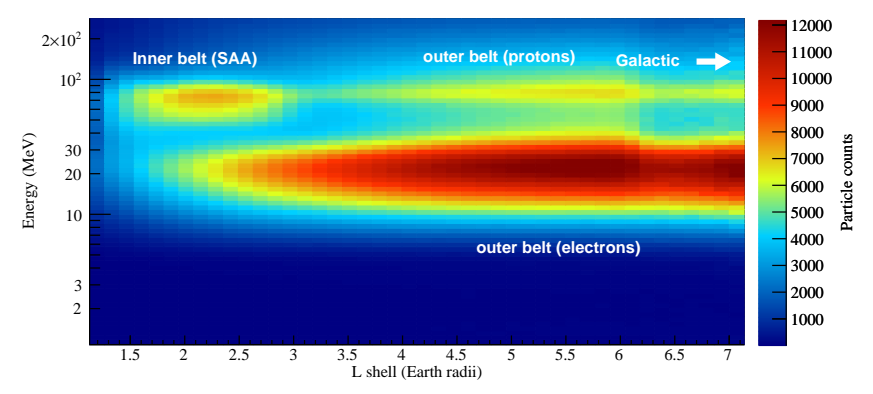

Figure 14. Different particle populations detected by HEPD as a function of L-shell and energy. lantic Anomaly obtained through SPENVIS ${ }^{3}$ model and HEPD data (August 2018), respectively.

Finally, Figure 16 reports the HEPD preliminary galactic proton flux compared to the Top-of-Atmosphere (TOA) theoretical fluxes for 2009 (blue), 2014 (magenta) and 2015 (red), together with LIS spectra (green). Precisely, good candidates have been selected to be compatible with the distribution of protons appearing in Figure 13, with the additional features of: 1) coming from outside the magnetosphere (this condition is fulfilled by setting a particle energy threshold two times greater than the local geomagnetic cutoff rigidity); 2) being fully contained inside the calorimeter tower, with only one-paddle hit in the trigger plane, and without any hit on any veto. Particular attention was paid to reject secondaries or events with spurious signals on more than one trigger paddle or LYSO crystal. The geometrical acceptance of the instrument has been estimated by a dedicated $4 \pi$ MonteCarlo simulation of protons in the range $1 \mathrm{MeV}-10 \mathrm{GeV}$. The flux presented in Figure 16 has to be considered preliminary, since the efficiency estimation has room for improvement, and associated errors are only statistical at the moment. Nonetheless, the flux appears consistent with the solar-cycle phase occurring on CSES launch (almost at the end of the $24^{\text {th }}$ solar cycle, approaching its solar minimum). Indeed, the minimum of the $23^{\text {rd }}$ solar cycle occurred in 2009, whereas the maximum of the $24^{\text {th }}$ took place in mid 2014. HEPD flux is, therefore, expected to lie between those of 2009 and 2014-2015, as confirmed by Figure 16.

\section{CONCLUSIONS}

CSES (China Seismo-Electromagnetic Satellite) is a Chinese-Italian space mission dedicated to monitoring variations of the electromagnetic field and waves, plasma parameters and particle fluxes induced by natural sources and artificial emitters in the near-Earth space. Austrian institutions contribute to the mission too.

The CSES satellite was launched from the Jiuquan Satellite Launch Center in the Gobi desert (Inner Mongolia) on Feb 2, 2018. The expected mission lifetime amounts to 5 years. The mission aims to study the existence of possible (temporal and spatial) correlations between the observation of iono-magnetospheric perturbations, as well as the precipitation of particles from the inner Van Allen belts, and the occurrence of seismic events. However, a careful analysis is needed in order to tell measurements possibly associated to earthquakes

\footnotetext{
3 https://www.spenvis.oma.be
} 

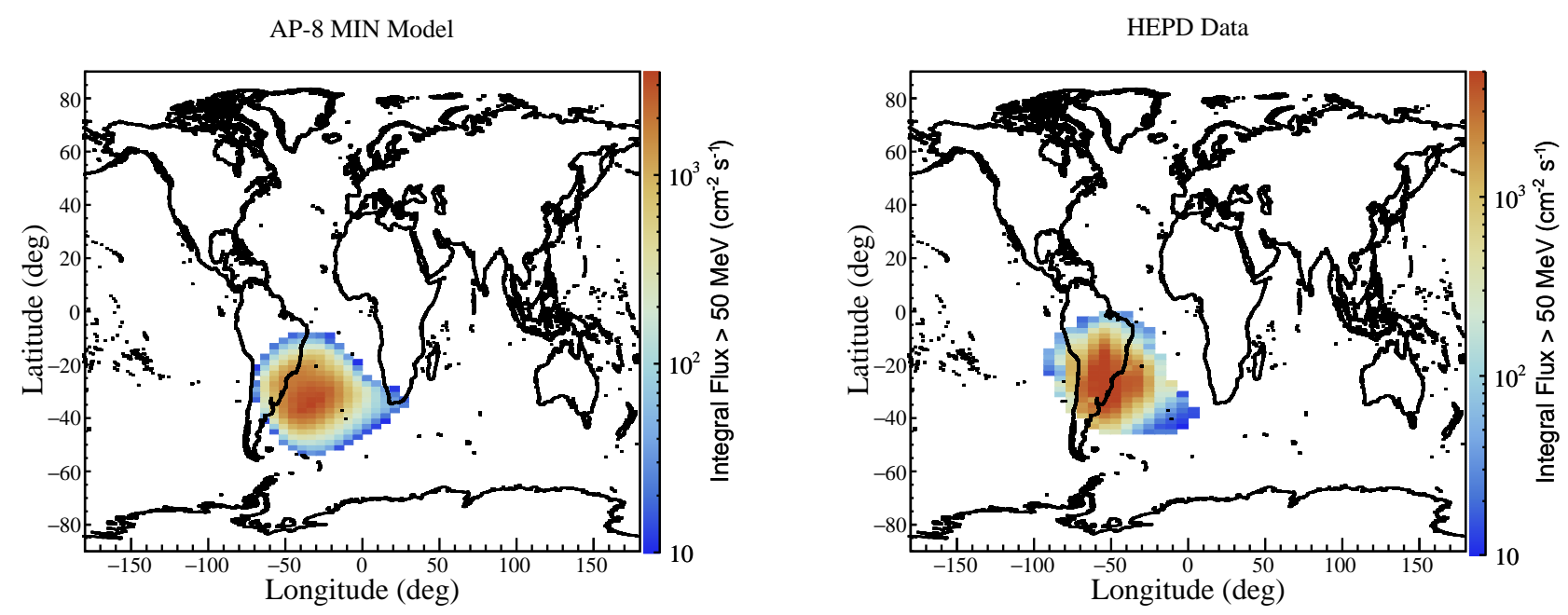

Figure 15. Comparison between trapped-proton geographical distributions inside the South Atlantic Anomaly obtained through the AP-8 MIN model (by the SPENVIS interface) and HEPD data (August 2018), respectively.

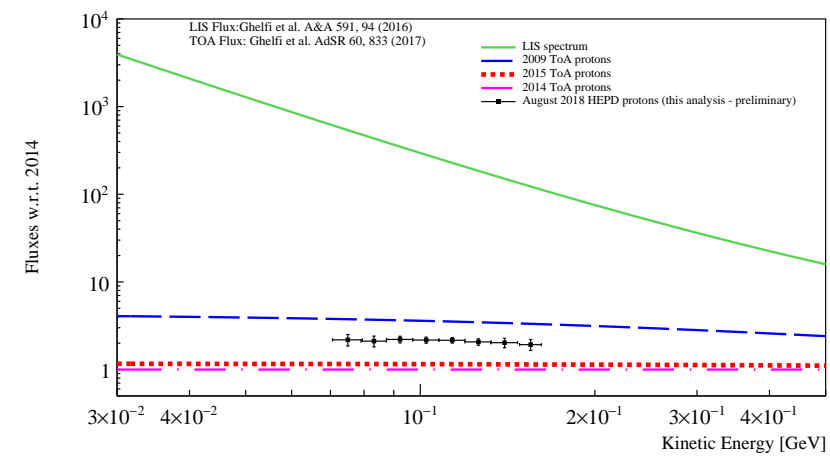

Figure 16. HEPD galactic proton flux (black squares) compared to Top-of-Atmosphere (TOA) theoretical fluxes for 2009 (blue dashed line), 2014 (magenta dashed-dotted line), 2015 (red dotted line), and LIS spectrum (green solid line).

apart from the large background generated in the geomagnetic cavity by solar activity and tropospheric electromagnetic emissions.

The Italian contribution to the mission includes the design and construction of the High-Energy Particle Detector (HEPD), aimed to detect electrons in the energy range between 3 and $100 \mathrm{MeV}$, and protons between 30 and $200 \mathrm{MeV}$, as well as light nuclei in the MeV energy window.

From the launch to the end of July 2018, CSES underwent a set of "commissioning tests", aiming to assess the good functioning of the instruments on board. All parameters were found nominal, both for the satellite and instruments, and the commissioning phase finished without remarks. As to HEPD, these months were used to find stable conditions under which to operate the instrument in flight, optimizing some working parameters.

Since August 2018, the science run of HEPD has started. In this article we have presented the first inflight collection of proton- and electron data: the particle rates are consistent with radiation models, as well as their distribution along the orbit.

Thanks to HEPD acceptance energy window, its orientation with respect to the Earth's magnetic-field lines, and satellite orbit, the instrument is capable of detecting different particle populations in space: solar particles, galactic particles, particles trapped in the inner and outer radiation belts including the SAA, and albedo particles. The first scientific runs of the instrument attest its good functioning all along the orbit, and confirm that HEPD is an optimal Space-Weather monitoring instrument.

\section{ACKNOWLEGDMENTS}

This work was supported by the Italian Space Agency in the framework of the Accordo Attuativo n. 2016-16-H0 Progetto Limadou Fase E/Scienza (CUP F12F1600011005).

\section{REFERENCES}

Adriani, O., et al., 2011, ApJ, 742, 102

Adriani, O., et al., 2013, ApJ, 765, 91

Adriani, O., et al., 2015a, ApJ, 810, 142
Adriani, O., et al., 2015b, ApJ Letters, 801, L3

Adriani, O., et al., 2015c, JGR, 120, 3728

Adriani, O., et al., 2015d, ApJ Letters, 799, L4 
Adriani, O., et al., 2016, Space Weather, 14, 210

Adriani, O., et al., 2017, La Rivista del Nuovo Cimento, 40, 10, 473

Alcaraz, J., et al., 2000, Phys. Let. B, 472, 215

Aleksandrin, S.Yu., 2003, Ann. Geophys., 21, 597

Aleshina, M.E., et al., 1992, Cosmic Research 30, 1, 79

Ambrosi, G., et al., 2018, Sci. China Technol. Sci., 61, 5, 643

Aguilar, M., et al., 2013, PRL 110, 141102

Balogh, A., et al., 2008, The Heliosphere through the Solar Activity Cycle, Springer

Battiston, R. \& Vitale, V., 2013, Nuclear Physics B

Proceedings Supplement, 244, 249

Bertello, I., et al., 2018 Ann. Geophys., 36, 1483

Biagi, P.F., et al., 2008, Nat. Hazards Earth Syst. Sci., 8, 1041

Cheng, B., et al., 2018, Sci. China Technol. Sci., 61, 5, 659.

Chmyrev, V.M., et al., 1997, J. Atmos. Sol. Terr. Phys., $59,9,967$

Cussac, T., et al., 2006, Plan. Sp. Sci., 54, 5, 413

Diego P., et al., 2017a , Adv. Space Res., 60, 10, 2206

Diego, P., et al., 2017b, IEEE Access, 5, 3824

Forbush, S.E., 1958, JGR, 63, 4, 651

Haggerty, D.K. \& Roelof, E.C., 2009, Proc. 8th Ann. Int'l Astrophys. Conf. AIP Conference Proceedings 1183, 3

Hayakawa, M., et al., 2010, J. Atmos. Sol. Terr. Phys., 72, 13,982

Jordanova, V.K., et al., 2006, JGR, 111, A11S10

Kintner, P.M., et al., 2002, Report of the LWS Geospace Mission Definition Team, NASA/TM 211613.

Kurnosova, L.T., et al., 1962, Plan. Sp. Sci., 9, 513

Lagoutte, D., et al., 2006, Plan. Sp. Sci., 54, 5, 428

Lario, D., et al., 2013, ApJ, 767, 41

Lorentzen, K. R., et al., 2000, JGR , 105, 5381

Mathews, T., \& Venkatesan, D., 1990, Nature, 345, 600

Meredith, N. P., et al., 2003, JGR, 108, A6, 1250

Molchanov, O. A., et al., 1993, Ann. Geophys., 11, 431
Molchanov, O.A. \& Hayakawa, M., 1998, JGR, 103, 17489

Moritz, J., 1972, Zeitschrift fuer Geophysik, 38, 701

Omura, Y., et al., 2010, JGR, 115, A07234

Parker, E.N., 1965, Plan. Sp. Sci., 13, 9

Parrot, M., \& Mogilevsky, M.M., 1989, Phys. Earth Planet. Int., 57, 86

Parrot, M., 1994, JGR, 99, A12, 23339

Parrot, M., 1995, Adv. Space Res., 15, 11, 27

Piersanti, M. \& Villante, U., 2016, JGR , 121, 6674

Piersanti, M., et al., 2017, Sol. Phys., 292, 169

Popova, I., et al., 2018, Entropy, 20, 691

Potgieter, M.S., 2014, Adv. Space Res., 53, 1415

Potgieter, M.S., et al., 2014, Solar Physics, 289, 1, 391

Scotti, V., et al., 2017, Nuclear and Particle Physics proceedings, 291, 118

Sgrigna, V., et al., 2005, J. of Atm. Sol. Terr. Phys., 67, 1448

Shen, Z.H., et al., 2018, Sci. China Technol. Sci., 61, 5, 634

Smart, D. F. \& Shea, M. A., 2000, Space Science Reviews, $93,1 / 2,305$

Smart, D. F. \& Shea, M. A., 2005, Adv. Space Res., 36, 10, 2012

Storini. M., et al., 2005, Adv. Space Res., 35, 416

Struminsky, A.B. \& Zimovetz I.V., 2010, Astronomy

Letters, 36, 6, 430

Summers, D. \& Thorne, R.M., 2003, JGR, 108, A4, 1143

Treiman, S.B., 1953, Phys. Rev., 91, 957

Tsyganenko, N. A. \& Sitnov, M. I., 2005, JGR, 110

Van Allen, J.A. \& Gangnes, A.V., 1950, Phys. Rev., 79, 51

Vellante, M., et al., 2014, JGR, 119, 2623

Vellante, M., et al., 2014b, XXXIth URSI General

Assembly and Scientific Symposium (URSI GASS), DOI: 10.1109/URSIGASS.2014.6929941

Yiyan, Z., et al., 2013, Geodesy and Geodynamics, 4, 4, 26

Zaharia, S., et al., 2006, JGR , 111, A11S14 


\section{APPENDIX}

In this Appendix additional details about technical aspects of the High-Energy Particle Detector single instruments are given and the structure of the HEPD electronics is described.

\section{A. THE HEPD SUB-DETECTORS}

\section{A.1. The Tracker Detector}

The tracking system of HEPD consists of 2 planes (called external and internal) of double-sided silicon micro-strip detectors, located at the top of the apparatus and separated by a 1-cm spacing. The signal is collected on both sides of the silicon wafer, with the implanted strips of one side orthogonal to those of the other, in order to provide a measurement of the $\mathrm{X}$ and $\mathrm{Y}$ coordinates of the incident ionizing particle.

In the HEPD tracker detector each silicon plane, with dimensions $213.2 \times 214.8 \mathrm{~mm}^{2}$ excluding the mechanics, is divided into 3 identical independent sections, called ladders, along the $\mathrm{X}$ axis. Such sections are named top, central and bottom, respectively, with the top one marked by the highest value of the $\mathrm{X}$ coordinate.

Each ladder contains two silicon sensors. The dimensions of the sensors, produced by FBK ${ }^{4}$, are $109.63 \mathrm{~mm} \times 77.58$ $\mathrm{mm} \times 0.3 \mathrm{~mm}$; the dimensions of the depleted volume (active area) are $106.63 \mathrm{~mm} \times 71.58 \mathrm{~mm} \times 0.3 \mathrm{~mm}$. The $\mathrm{p}^{+}$ side contains 767 implantation strips, with a $182-\mu \mathrm{m}$ pitch. The ohmic side contains $1151 \mathrm{n}^{+}$implant strips, alternated with $\mathrm{p}^{+}$blocking strips to minimize the effect of the surface charge present on the ohmic side. An assembled silicon tracker plane is shown in figure 17 , left.

Modules are wire-bonded to each other on $\mathrm{p}^{+}$strips, each of them read-out with a dedicated channel. To save read-out channels, $\mathrm{n}^{+}$strips are grouped in three for read-out, with a degeneracy (Y direction) solved exploiting the information from the trigger plane. The read-out strips are directly connected with the read-out electronics by means of $\mathrm{AC}$ pads (i.e., a capacitor connects the strips to the read-out amplifiers), while the non read-out strips (floating strips) allow to increase the spatial resolution, inducing signal on the adjacent read-out ones via capacitive coupling.

\section{A.2. The Trigger Plane}

The trigger system is made of one thin layer of plastic scintillator $\left(20 \times 18 \times 0.5 \mathrm{~cm}^{3}\right)$ divided into 6 segments (or paddles) with dimensions $20 \times 3 \times 0.5 \mathrm{~cm}^{3}$, each one read by two Hamamatsu Photo-Multiplier Tubes (PMTs). The plane is located below the tracker system and performs the following tasks:

- fast trigger-signal generation to start and synchronize data acquisition for the whole apparatus;
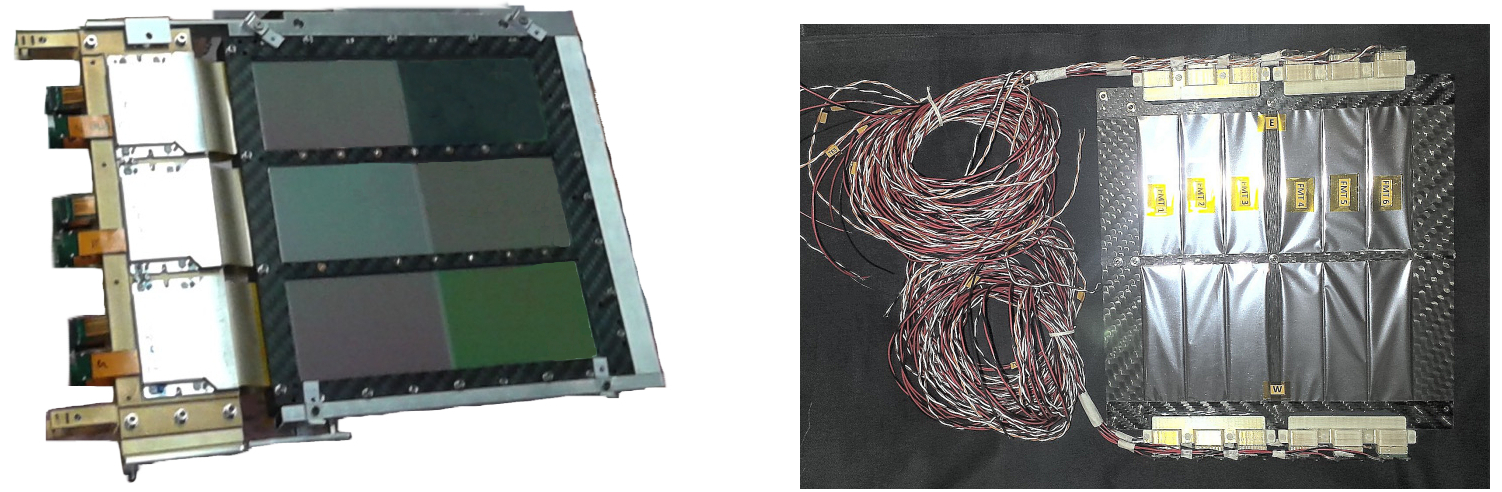

Figure 17. Left: An assembled plane of silicon detecting units. Right: A picture of the segmented trigger plane.

\footnotetext{
${ }^{4}$ Fondazione Bruno Kessler: https://www.fbk.eu
} 


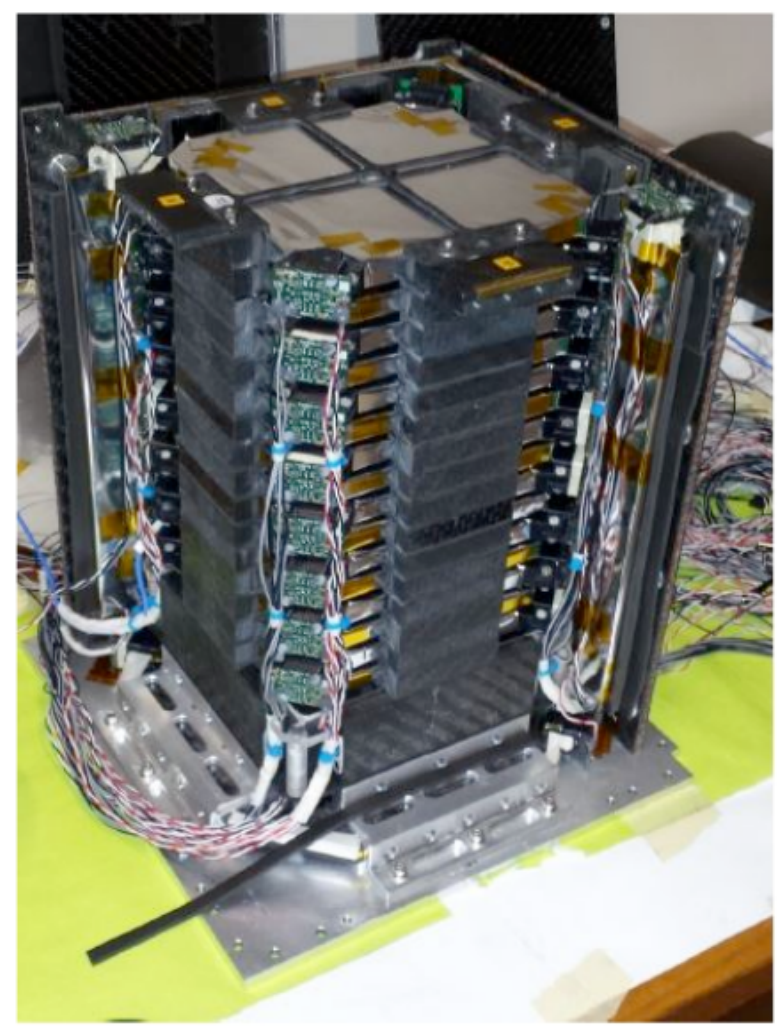

Figure 18. A picture of the assembled scintillator tower.

- resolution of the ambiguity of the Y-coordinate position due to the daisy-chaining of 3 readout strips to the same readout channel on the ohmic side of the silicon sensors;

- possible secondary determination of the ionization energy loss produced in the layer, in addition to the more precise measurement obtained by the tracker;

- possible rejection of events characterized by more than one paddle hit (multi-particle events).

To generate the trigger signal, the coincidence between a signal on at least one of the trigger paddles and the signal from at least one additional calorimeter plane is required. A picture of the trigger plane can be seen in figure 17, right.

\section{A.3. The Calorimeter}

The range calorimeter of the HEPD detector consists of two different parts. The first one, on the top and immediately below the trigger plane, is made of 16 planes of plastic scintillator. Each plane has dimensions $15 \times 15 \times 1 \mathrm{~cm}^{3}$ and is read out by two PMTs, placed at two opposite corners of the plane. The bottom part of the calorimeter consists of a $3 \times 3$ matrix of LYSO inorganic-scintillator crystals, for a resulting plane with total dimensions $15 \times 15 \times 4 \mathrm{~cm}^{3}$.

The mechanical stability of the system is assured by a support structure, which holds the detector in place and prevents any damage during the launch phase. The scintillator counters are arranged in a vertical stack and positioned by means of a carbon fiber frame, each one mounted on top of another, in such a way to form a rigid tower where each counter is separated by the adjacent ones (see picture in figure 18). Layers of relatively soft open-cell Poron, placed between the counters and the carbon fiber, prevent any stress and shock possibly transferred to the counters themselves, such to avoid damages. The high rigidity of the system provides a great safety margin against stresses and vibration shocks.

The aim of the calorimeter is the measurement of the energy deposition and range of the impinging particles. The presence of the LYSO bottom layer increases the operational energy range of the detector by means of a larger matter thickness with high density. 


\begin{tabular}{|l|l|}
\hline PROPERTIES & EJ-200 \\
\hline Light Output (\% Anthracene) & 64 \\
\hline Efficiency (photons $/ 1 \mathrm{MeV} \mathrm{e}{ }^{-}$) & 10 \\
\hline$\lambda$ of Maximum Emission $(\mathrm{nm})$ & 425 \\
\hline Light Attenuation Length $(\mathrm{cm})$ & 380 \\
\hline Rise Time $(\mathrm{ns})$ & 0.9 \\
\hline Decay Time $(\mathrm{ns})$ & 2.1 \\
\hline Density $\left(\mathrm{g} / \mathrm{cm}^{3}\right)$ & 1.023 \\
\hline Temperature Range & $-20^{\circ} \mathrm{C}$ to $60^{\circ} \mathrm{C}$ \\
\hline
\end{tabular}

Table 2. Some properties of the EJ-200 plastic scintillator.

\section{A.3.1. Plastic Scintillators}

The scintillator material selected for all the plastic planes (trigger, calorimeter, veto system) is the EJ-200 by Eljen Technology, which combines long optical attenuation length and fast timing. It consists of an organic polymer (Polyvinyltoluene, refractive index 1.58), which is luminescent when irradiated by ionizing particles. Some properties of the EJ-200 are reported in Table 2, while figure 19 (right) shows its emission spectrum.

The four corners of every scintillator plane in the calorimeter have been cut in order to create room to place the PMTs. Each plane contains two PMTs, placed at two opposite corners. The external walls of the planes, with the exception of the entrance windows of the PMTs, are optically insulated by means of a thick mylar foil with about $98 \%$ reflectivity index. This foil reflects back the fraction of scintillation light that escapes the plane, thus increasing energy resolution.

\section{A.3.2. LYSO Matrix}

The bottom part of the calorimeter is made of a LYSO (Cerium-doped Lutetium Yttrium Orthosilicate) inorganic scintillator. This is a high-density material $\left(\mathrm{d}=7.3 \frac{\mathrm{g}}{\mathrm{cm}^{3}}\right)$, which, combined with its 4-cm thickness, allows to increase the operational energy range of the energy detector.

The LYSO layer is formed by a $3 \times 3$ matrix (see figure 19, right), where each crystal has dimensions $4.8 \times 4.8 \times 4 \mathrm{~cm}^{3}$ and is read out by a single PMT placed in the bottom side (the side opposite to the trigger plane). These crystals offer the advantage of a high light output, quick decay time and excellent energy resolution. The peak of the emission spectrum is at $428 \mathrm{~nm}$; this makes it possible the use of the same PMTs adopted for the plastic-scintillator planes as read-out devices.

\section{A.3.3. Photo Multiplier Tubes}

The PMT model chosen to read all scintillator counters out, is the R9880-210, manufactured by Hamamatsu. As can be seen in figure 20 (a), the quantum efficiency spectrum (i.e., the ratio between the number of output electrons
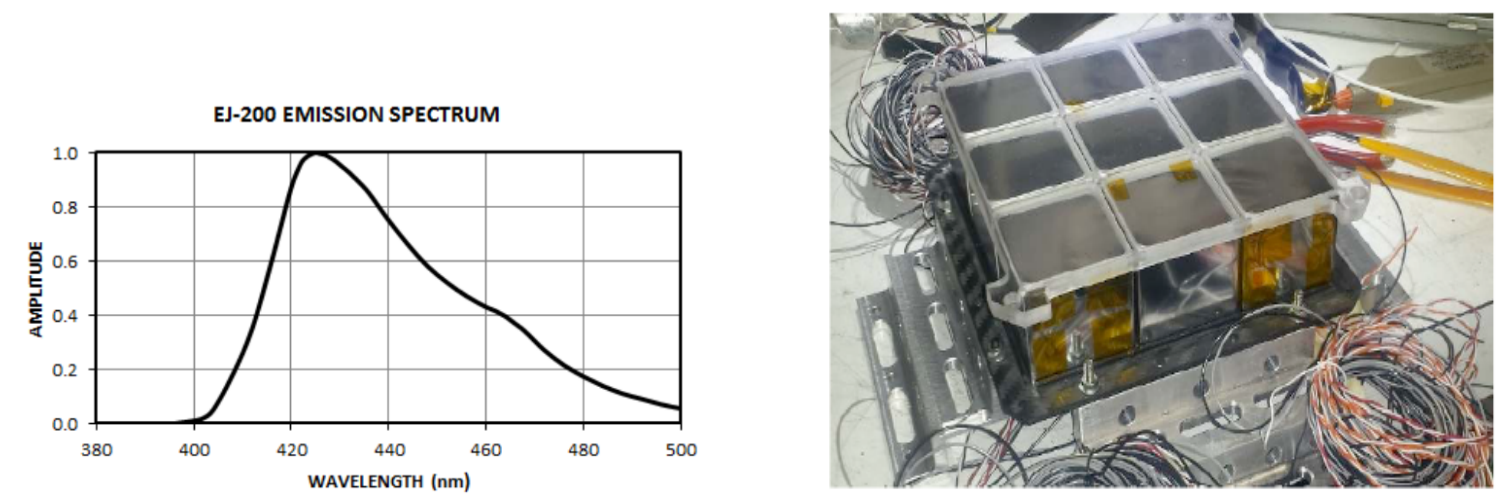

Figure 19. Left: Emission spectrum of the EJ-200 plastic scintillator. Right: A picture of the LYSO matrix plane. 

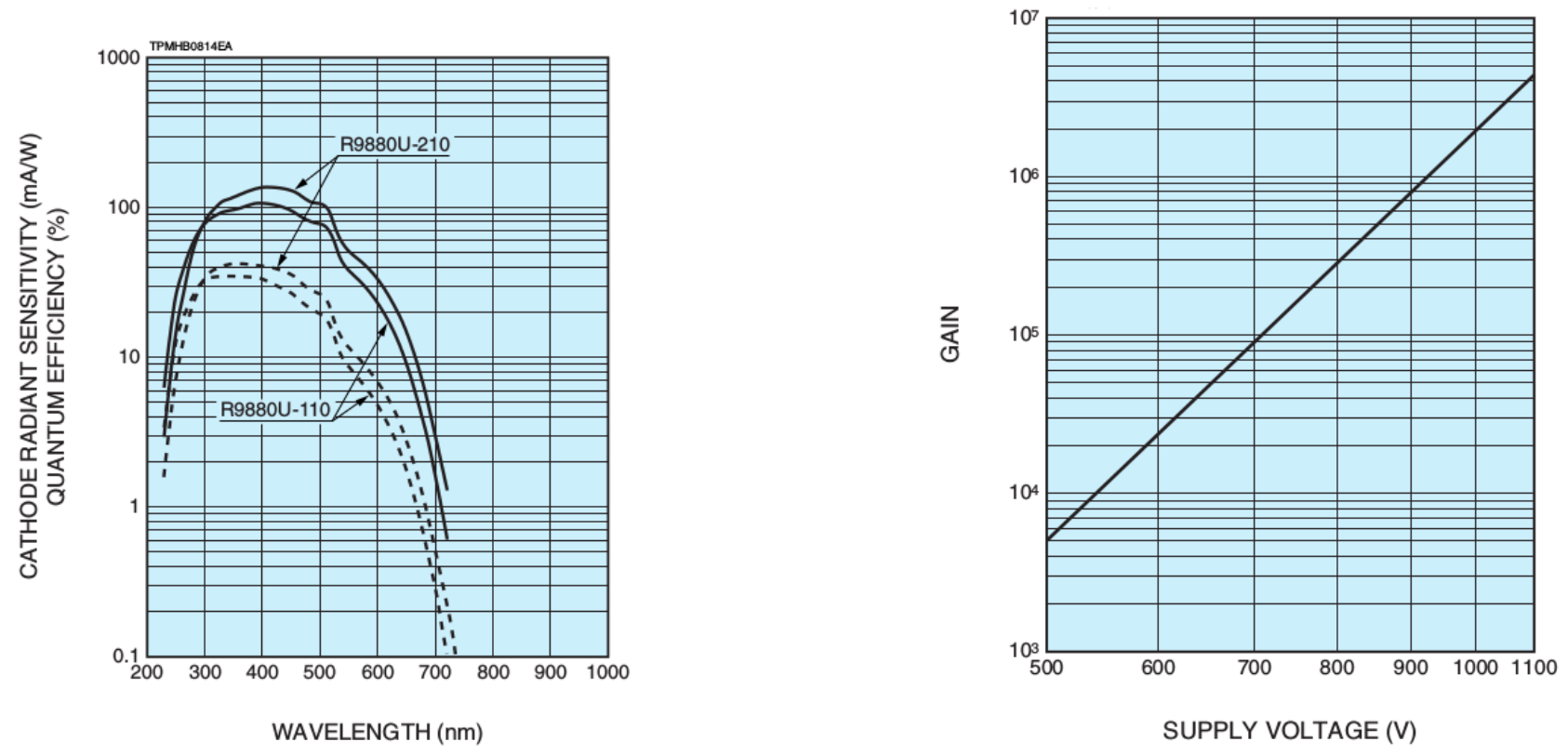

Figure 20. a) PMT quantum efficiency, and b) gain.

and incident photons) matches the light emission band characteristic of the scintillator EJ-200 with a peak at 425 nm. Furthermore, the R9880-210 is a small device (with a cylindrical shape and an 8-mm-diameter effective area for the entrance window) with a small weight and an operating temperature in the range $-80^{\circ} \mathrm{C} \div+50^{\circ} \mathrm{C}$, all important parameters to be taken into account for a space-borne apparatus.

Considering the supply voltage between 750 and $900 \mathrm{~V}$ provided by the HEPD power supply system, the gain obtained by the R9880-210 is in the order of $10^{5}-10^{6}$ (see figure 20 (b) ).

The interface between any PMT and scintillator plane is obtained through a soft optical pad.

\section{A.4. The Veto System}

The veto system includes five thin EJ-200 plastic scintillators that completely surround the calorimeter. The veto counters are placed inside a volume created by the calorimeter structure and carbon-fiber honeycomb plates located externally. A Poron thickness, placed all around the scintillators, prevents any stress and shock possibly transferred to the counters.

The veto sub-detector is fundamental in rejecting any type of background caused by out-of-acceptance events. In particular, the system is designed for:

- the identification and rejection of particles that do not cross the two tracking planes, but generate a trigger signal (for example, by laterally entering the apparatus);

- the rejection of secondary particles produced inside the calorimeter;

- the identification of not fully contained events.

All planes of the veto system are 5-mm thick and read out by two R9880-210 Hamamatsu PMTs. Four planes are located at the lateral sides of the calorimeter and the fifth one is below the LYSO layer, to reject particles that are not fully contained within the calorimeter, or up-going particles.

\section{B. HEPD ELECTRONICS}

The most important requirement for a space experiment electronics system is to assure high reliability over the whole duration of the mission ( 5 years for CSES-Limadou), and low power consumption (power budget $<43 \mathrm{~W}$ for HEPD). 
The probability of a failure increases with the overall operating time and with the total number of performed power cycles. Redundancy is the best way to limit the consequences of permanent failures in the electronics subsystem. Therefore, each electronic board has been duplicated on a physical board, which hosts two identical copies of the same electronics (main and spare side). In this configuration (cold redundancy) the spare side is available in case of failure of the main side. Hot and cold sides are completely independent of each other, and cannot be powered at the same time. A second level of redundancy has also been applied to some important components for each board. The drawback of this design is clearly the multiplication of the number of components, connections and wires.

Moreover, a possible mechanical damage of a board induced by strong accelerations during the launch phase has been taken into account with an accurate FEM analysis. Mechanical qualification tests for the single board and for the whole apparatus have been carried out in qualified facilities before the launch.

In this section the most significant characteristics of HEPD electronics boards are illustrated (Scotti et al. 2017). A scheme of the HEPD ELectronics Subsystem (ELS) is shown in figure 21.

\section{B.1. Power Control Board and High-Voltage Board}

The power control board (also named control low-voltage board) provides the 'digital' voltages for all other components of the ELS. The board is divided into the usual hot/cold sides, and a common section that includes the connector for a direct link with the satellite. The platform uses this arrangement to send the direct STAND_BY_ON, STAND_BY_OFF and RESET hardware commands to HEPD. The logic of the board is implemented on an Actel FPGA (A3P125).

The High-Voltage board is instead responsible for the voltage output for the HEPD detectors. It comprises a scintillator section, formed by $10 \mathrm{HV}$ modules (which provide a voltage between 750 and $900 \mathrm{~V}$ ), and a silicon section, formed by $2 \mathrm{HV}$ modules (which provide $60 \mathrm{~V}$ ), one for each plane.

\section{B.2. Trigger Board}

The tasks of the trigger board are summarized as follows:

- acquisition of the 63 PMTs by means of the EASIROC ASICs;

- analog-to-digital conversion of the PMT signals, and their transfer to the DAQ board;

- management of the trigger configurations and generation of the trigger pulse;

- generation of the test trigger pulse for calibration purposes;

- measurement of the dead time and live time of the apparatus;

- measurement of the rate meter for each PMT and for each trigger configuration.

To provide all these features, each side (hot/cold) of the board relies on an FPGA (Actel ProASIC A3PE1500), two EASIROC chips and four 12-bit ADCs.

\section{B.3. Data AcQuisition Board}

The Data AcQuisition board (DAQ) provides the following functionalities:

- an interface with the front-end electronics of the silicon planes;

- processing and digitization (by dedicated ADCs) of the analog signal coming from the silicon plane front end;

- online calibration of the silicon detector;

- acquisition of the PMT data coming from the trigger board;

- compression of the data, in order to reduce the size of the events; 


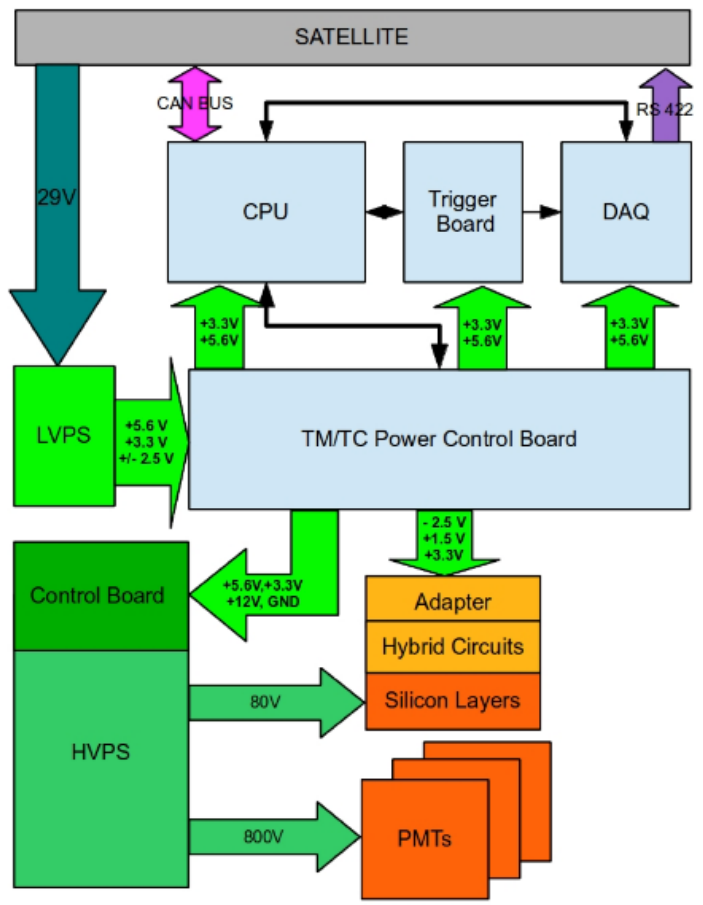

Figure 21. A general scheme of HEPD electronics and power supply subsystems. Communication and power lines between the boards and towards the satellite are shown as well.

- formatting and transmission of the data to the satellite via the RS-422 interface (scientific data link).

To support all these functionalities, the board is mainly composed of:

- a Digital Signal Processor (ADSP-2189M) for all the computing operations;

- 2x FPGAs (Main and Download);

- 2x non-volatile Ferroelectric Random Access Memories (FRAM) for the permanent storage of the code to be executed by the DSP and other important data required at the boot of the board;

- a Dual-Port Random Access Memory (DPRAM) used for the data handling between the Main FPGA and DSP;

- a Static Random Access Memory (SRAM) used as a FIFO ${ }^{5}$ for the data to be transferred towards the satellite;

- 8x 12-bit ADCs for the digitization of the silicon detector data.

The operating frequency of the board was set at $48 \mathrm{MHz}$, as the best compromise between the requirement of fast operations and not too high a power consumption.

Ferroelectric RAMs are random access memories that use a ferroelectric layer instead of a dielectric one to achieve the non-volatility ${ }^{6}$.

\section{B.4. $C P U$ Board}

The CPU board is the digital subsystem that controls the detector status and communicates with the platform of the satellite via the CAN bus interface. The board manages the following functionalities:

- communication with the satellite computer $(\mathrm{OBDH})$ via the 2x CAN bus interface (nominal and redundant);

${ }^{5}$ Acronym for First In, First Out. It is a method for organizing data buffer, where the oldest (first) entry is processed first.

${ }^{6}$ A non-volatile memory is a type of memory that can retrieve stored information even after having been powered off. 
- management of the Power Control board, High-Voltage control board, Trigger board and DAQ board, via internal slow control link bus;

- management of system diagnostic routines and system configuration.

The main components of the board are:

- an FPGA (Actel ProAsic3 A3PE1500) for safe boot management and implementation of the slow control link;

- a digital signal processor ADSP-2189M;

- 2x CAN bus transceivers and 2x CAN bus controllers SJA1000T;

- a Read-Only EEPROM and a Read-Write FRAM used to store the application code for the digital signal processor. 